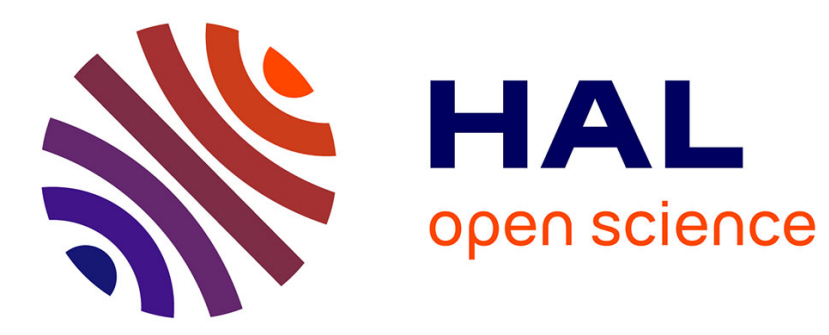

\title{
Modélisation de matériaux chiraux à structures hétérogènes (modèle MTWC) : théorie, validations expérimentales et applications \\ F. Mariotte, B. Sauviac, J. Héliot
}

\section{- To cite this version:}

F. Mariotte, B. Sauviac, J. Héliot. Modélisation de matériaux chiraux à structures hétérogènes (modèle MTWC) : théorie, validations expérimentales et applications. Journal de Physique III, 1995, 5 (10), pp.1537-1564. 10.1051/jp3:1995209 . jpa-00249400

\section{HAL Id: jpa-00249400 https://hal.science/jpa-00249400}

Submitted on 1 Jan 1995

HAL is a multi-disciplinary open access archive for the deposit and dissemination of scientific research documents, whether they are published or not. The documents may come from teaching and research institutions in France or abroad, or from public or private research centers.
L'archive ouverte pluridisciplinaire HAL, est destinée au dépôt et à la diffusion de documents scientifiques de niveau recherche, publiés ou non, émanant des établissements d'enseignement et de recherche français ou étrangers, des laboratoires publics ou privés. 
Classification

Physics Abstracts

$41.10-41.10 \mathrm{~F}-41.10 \mathrm{H}$

\title{
Modélisation de matériaux chiraux à structures hétérogènes (modèle MTWC) théorie, validations expérimentales et applications
}

\author{
F. Mariotte, B. Sauviac et J. Ph. Héliot \\ Commissariat à l'Energie Atomique, CEA-CESTA B.P. 2, 33114 Le Barp, France
}

(Reçu le 24 février 1995, révisé le 20 juin 1995, accepté le 13 juillet 1995)

Résumé. - Après un bref rappel du principe de la chiralité, cet article présente une modélisation des propriétés effectives des matériaux hétérogènes à inclusions chirales métalliques : calcul de la permittivité, perméabilité et coefficient de chiralité du composite en fonction de la fréquence. Les résultats théoriques sont validés, pas à pas, par des mesures effectuées sur des composites chiraux de natures différentes. L'application de tels matériaux à la conception de matériaux absorbant les ondes électromagnétiques est ensuite envisagée. Les inclusions chirales semblent offrir la possibilité de régler l'impédance à l'interface air-milieu absorbant permettant ainsi de concevoir des absorbants micro-ondes plus performants en terme d'atténuation ou de largeur de bande. L'optimisation des caractéristiques des matériaux pour obtenir des performances données restent néanmoins très délicate.

\begin{abstract}
After a brief overview of the concept of electromagnetic chirality, this paper deals with a numerical simulation of isotropic composites with metallic chiral inclusions: computations of permittivity, permeability and chirality parameter as functions of frequency are presented. The theoretical results are, step by step, compared with measurements of chiral composites at microwave frequencies. The application of such media in Radar Cross-Section (RCS) management and control is discussed. The introduction of chiral inclusions seems to make impedance matching possible and may lead to attractive shields with lower reflectivity and larger bandwidth. However the optimization of material characteristics necessary to get a specific absorber remains a difficult task.
\end{abstract}

\section{Introduction}

Les absorbants micro-ondes sont en général des matériaux homogènes isotropes à pertes diélectriques ou magnétiques. Ils sont utilisés dans des architectures monocouches (écrans de Dällenbach), bicouches (écrans de Salisbury par exemple) ou multicouches, suivant les performances demandées: absorption sur une large bande ou à une fréquence donnée. Depuis quelques années, on observe une utilisation croissante des matériaux hétérogènes isotropes ou anisotropes. Ils 


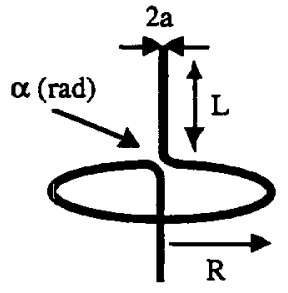

a)

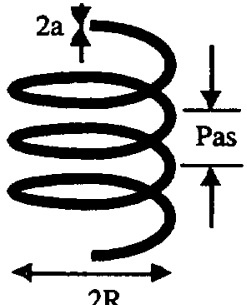

b)

Fig. 1. - Quelques objets chiraux : a) hélice modèle ou hélice de Jaggard. b) hélice à 4 tours à enroulement à droite.

[Two chiral objects: a) canonical or Jaggard helix. b) 4 turns helix.]

consistent en une dispersion (aléatoire ou en réseau) d'inclusions (charges métalliques, diélectriques ou magnétiques) de formes variées (bâtonnets, sphères, disques...) dispersées dans une matrice polymère ou céramique. Très récemment les recherches se sont portées sur de nouveaux types de matériaux hétérogènes : les matériaux chiraux. Dans ce cas, les inclusions sont le plus souvent des hélices métalliques ou céramiques. Les composites ainsi constitués sont décrits par des équations constitutives où les champs électrique et magnétique sont couplés.

Afin de pouvoir étudier ces nouveaux matériaux et de dégager leurs applications potentielles, il est indispensable de disposer d'une connaissance précise des caractéristiques électromagnétiques de matériaux chiraux obtenus par insertion de particules chirales macroscopiques, qu'il s'agisse d'hélices diélectriques, magnétiques ou conductrices, dans une matrice, et de préciser les domaines de variation de ces caractéristiques en fonction de la fréquence. Nous nous proposons donc de calculer les propriétés effectives de composites intégrant des inclusions chirales métalliques dans des matrices de caractéristiques électromagnétiques données. Disposant de cette modélisation, nous étudions une application particulière pour de tels matériaux : les absorbants micro-ondes.

\section{Quelques rappels sur la chiralité}

Les matériaux chiraux existent à l'état naturel ; on peut citer par exemple le quartz ou les cristaux liquides ferroélectriques. Toutefois dans ce document, on entend par "matériau chiral", un matériau hétérogène constitué de charges chirales réparties de façon isotrope dans un liant (appelé aussi milieu hôte). La notion de chiralité est une notion purement géométrique. Par définition un objet est chiral si on ne peut pas le superposer par translation ou rotation à son image spéculaire dans un miroir plan [1]. Dans la suite de cet article, les charges chirales sont des microstructures dissymétriques de taille millimétrique (hélices à un ou plusieurs tours par exemple, Fig. 1). Ces inclusions peuvent être diélectriques, magnétiques ou conductrices et leur dimension n'est pas limitée. Si leur taille reste faible devant la longueur d'onde, on peut parler de milieu effectif et définir une permittivité, une perméabilité et un coefficient de chiralité pour le composite. Dans le cas contraire, les inclusions chirales n'étant plus petites devant la longueur d'onde, le matériau chiral peut être assimilé à un ensemble de structures diffractantes et diffusantes et on ne peut plus parler de milieu effectif. L'objectif des travaux 
théoriques décrits par la suite est de relier les propriétés effectives du composite chiral aux paramètres suivants : géométrie, nature et concentration des inclusions chirales (hélices) et caractéristiques radioélectriques du milieu hôte.

Les milieux chiraux sont décrits par des équations constitutives, qui dans le formalisme que nous avons choisi d'employer [2], s'écrivent sous la forme suivante (convention $\exp \{-j \omega t\}$ ):

$$
\begin{aligned}
& \mathbf{D}=\varepsilon_{\mathrm{c}} \mathbf{E}+j \chi_{\mathrm{c}} \mathbf{H} \\
& \mathbf{B}=\mu_{\mathrm{c}} \mathbf{H}+j \chi_{\mathrm{c}} \mathbf{E}
\end{aligned}
$$

où $\varepsilon_{\mathrm{c}}$ et $\mu_{\mathrm{c}}$ désignent les permittivité et perméabilité du matériau chiral et $\chi_{\mathrm{c}}$ représente le paramètre de chiralité qui décrit le couplage entre les champs électrique et magnétique, dû à la forme chirale des objets inclus dans le matériau. De tels milieux présentent des propriétés de biréfringence et de dichroïsme circulaires. Ils possèdent deux modes propres de propagation, une onde polarisée circulaire droite et une onde polarisée circulaire gauche [3]. Les équations (1) et (2) sont un cas particulier des relations constitutives des milieux bi-anisotropes, dont les propriétés électromagnétiques ont été étudiées par Kong [4]. Il faut rappeler qu'il existe différents formalismes pour décrire les milieux chiraux $[3,5]$. Ils sont tous équivalents et l'on peut aisément trouver des relations pour passer d'un formalisme à l'autre $[3,6]$. Toutefois, les paramètres effectifs ont des significations différentes selon le type de relations utilisées ; on notera simplement que dans les équations constitutives que nous avons choisies précédemment, la permittivité et la perméabilité ont strictement la même signification que pour des matériaux diélectriques ou magnétiques isotropes.

\section{Modélisations de matériaux chiraux à structures hétérogènes}

Dans cette partie, nous proposons un calcul des propriétés effectives de composites intégrant des inclusions chirales métalliques réparties de façon aléatoire dans un liant à pertes de caractéristiques électromagnétiques données. Il est à noter que la littérature antérieure à 1993 mettait en évidence une absence de modélisation efficace de tels composites. Parmi les travaux existants, on peut citer ceux de Jaggard et al. [1] qui ont développé un modèle macroscopique de l'interaction d'une onde électromagnétique avec une collection d'hélices petites devant la longueur d'onde (approximation quasi-statique), réparties de façon isotrope dans le vide. Ces travaux ont été repris et complétés par Zouhdi et al. en 1992 [7]. Ce modèle présente des incohérences avec certains principes physiques, notamment avec le principe de réciprocité [8]. Par la suite, Lakhtakia et al. [3] ont assimilé une hélice à une série de petites sphères diélectriques disposées de façon hélicoïdale : chaque sphère ayant un moment dipolaire $\mathbf{p}$, les moments dipolaires électrique et magnétique équivalents à la collection de sphères sont calculés. Sihvola et al. [9] ont développé une loi de mélange de type Maxwell Garnett pour un ensemble d'inclusions chirales sphériques noyées dans un liant, mais dans ce modèle les auteurs ne précisent pas comment calculer les paramètres effectifs des inclusions chirales sphériques. Plus récemment, des approches analytiques $[8,10-12]$ ont été utilisées pour modéliser les composites chiraux. Ces modèles ont été validés expérimentalement avec succès ; toutefois ils ne peuvent prendre en compte que des hélices modèles parfaitement conductrices (Fig. 1a). Mariotte et al. [13] ont étudié de façon exhaustive l'interaction d'une onde électromagnétique avec une inclusion chirale de géométrie quelconque, métallique ou diélectrique. Enfin, plusieurs modèles numériques ont été développés récemment [14-22]. On peut citer notamment celui de Brewitt-Taylor (modélisation de composites à inclusions métalliques noyées dans un liant sans perte) [17]), et celui de Whites basé sur une méthode Monte-Carlo [18]. 
Le modèle que nous proposons comporte deux étapes [8] : tout d'abord nous étudions les propriétés d'une inclusion, en l'occurrence ici une hélice à un ou plusieurs tours, puis nous déterminons les paramètres effectifs du matériau composite constitué d'une collection d'hélices noyées dans un milieu hôte à pertes en utilisant une loi de mélange de type Maxwell Garnett.

\section{1. ÉTUdE D'UNE INCLUSION CHIRALE}

3.1.1. Principe. - La première étape consiste donc à étudier une inclusion chirale. Il s'agit de calculer l'interaction d'une hélice avec une onde électromagnétique. Pour ceci nous utilisons la version filaire du code numérique ARLENE développé au CEA/CESTA [23] : elle est basée sur une méthode intégrale couplée avec l'approximation filaire, ce qui permet de remplacer la discrétisation $2 \mathrm{D}$ par des éléments finis $1 \mathrm{D}$ et donc d'accélérer considérablement les calculs.

Le code ARLENE s'intéresse au problème de la diffraction d'une onde électromagnétique incidente $\mathbf{E}_{\text {inc }}$ par un obstacle conducteur de conductivité donnée $\sigma$, occupant un volume borné $\Omega$ de frontière $\Gamma$. Le champ électromagnétique total $(\mathbf{E}, \mathbf{H})$ vérifie en tout point de l'espace les équations de Maxwell et les conditions de rayonnement de Sommerfeld. $\Omega$ n'étant pas un conducteur parfait, $\mathbf{E}$ et $\mathbf{H}$ ne sont pas nuls dans $\Omega$, la continuité de la composante tangentielle de $\mathbf{E}$ à travers $\Gamma$ s'écrit :

$$
\mathbf{E}-\mathbf{n}(\mathbf{n} \cdot \mathbf{E})=Z_{\mathrm{c}}(\mathbf{n} \times \mathbf{H})
$$

où $Z_{\mathrm{c}}$ est l'impédance de surface de l'obstacle conducteur et $\mathrm{n}$ la normale sortante à la surface frontière $\Gamma$. On suppose que cet obstacle est un conducteur filaire. Le champ électrique incident induit à la surface du fil métallique une densité de courant $\mathbf{J}_{s}$, qui à son tour, rayonne un champ diffracté $\mathbf{E}_{\mathrm{d}}$ vérifiant l'équation vectorielle d'Helmholtz suivante :

$$
-\Delta \mathbf{E}_{\mathrm{d}}-\beta^{2} \mathbf{E}_{\mathrm{d}}=j \omega \mu \mathbf{J}_{\mathbf{s}}+j \frac{1}{\omega \varepsilon} \nabla\left(\nabla \cdot \mathbf{J}_{\mathrm{s}}\right)
$$

avec $\beta=\omega \sqrt{\varepsilon \mu}, \omega$ étant la pulsation de l'onde incidente et $\varepsilon, \mu$ les caractéristiques radioélectriques du milieu entourant l'obstacle. Pour utiliser l'approximation filaire, l'objet doit vérifier les deux conditions suivantes:

- deux de ses dimensions sont très petites devant la troisième,

- le rayon de courbure suivant la plus grande dimension est suffisamment grand.

Le cas usuel est le cylindre rectiligne de section circulaire de rayon $a$ et de longueur $L$ tel que : $a \ll L$.

Dans la version filaire du code ARLENE, nous considérons donc un fil cylindrique $\Omega$, de grand rayon de courbure, de section circulaire de rayon $a$ et de longueur $L$ (Fig. 2). Compte tenu de la géométrie du problème, on utilise les coordonnées cylindriques $(\rho, \theta, s)$. L'approximation filaire consiste à dire que le courant $\mathbf{J}_{s}(s, \theta)$ est indépendant de $\theta$ et parallèle à la tangente à l'axe $\Sigma$ du cylindre, ce qui se traduit, au point d'abscisse curviligne $s$, par la relation :

$$
\mathbf{J}_{\mathbf{s}}(s, \theta)=\left|\mathbf{J}_{\mathbf{s}}(s)\right| \boldsymbol{\tau}(s)=J_{\mathbf{s}}(s) \boldsymbol{\tau}(s)
$$

où $\tau(s)$ est le vecteur unitaire porté par $\Sigma$.

La solution élémentaire associée à l'équation (4) peut s'écrire :

$$
G(R)=\frac{\exp \{j \beta R\}}{4 \pi R}
$$




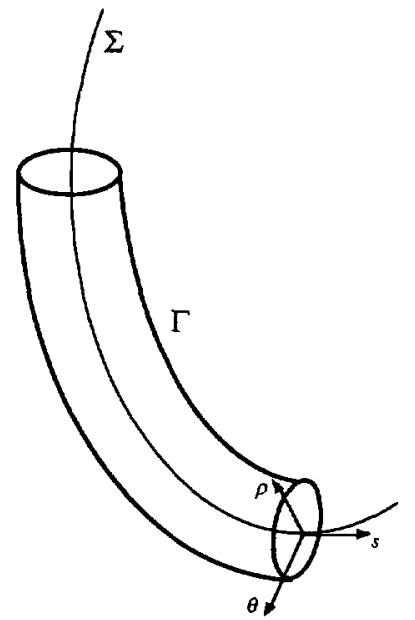

a)

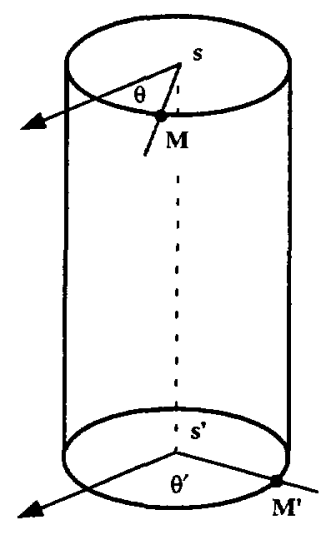

b)

Fig. 2. - Géométrie filaire. a) Système de coordonnées cylindriques pour un objet filaire de rayon de courbure quelconque. $\Sigma$ est la trace de l'axe du cylindre. b) Cas où les points $M$ et $M^{\prime}$ sont proches. [Thin wire geometry.]

Le champ diffracté $\mathbf{E}_{\mathrm{d}}$ s'exprime alors sous forme intégrale comme la convolution de $G$ avec le second membre de l'équation (4) :

$$
\mathbf{E}_{\mathrm{d}}(r)=\jmath \omega \mu \int_{\Gamma} G\left(\left|r-r^{\prime}\right|\right) \mathbf{J}_{\mathbf{s}}\left(r^{\prime}\right) \mathrm{d} r^{\prime}+\jmath \frac{1}{\omega \varepsilon} \nabla \int_{\Gamma} G\left(r-r^{\prime}\right)\left(\nabla \cdot \mathbf{J}_{\mathbf{s}}\left(r^{\prime}\right)\right) \mathrm{d} r^{\prime}
$$

où $r^{\prime}$ est un point à la surface de l'obstacle et $r$ un point quelconque dans l'espace. Finalement, on peut déterminer en tout point $r$, le champ électrique total $\mathbf{E}$ à partir de $\mathbf{J}_{\mathbf{s}}$ par :

$$
\mathbf{E}(r)=\mathbf{E}_{\mathrm{inc}}(r)+\mathbf{E}_{\mathrm{d}}(r)
$$

En utilisant un champ de vecteurs tests $\mathbf{J}^{\prime}$, tangents à $\Gamma$ et dirigés suivant l'axe du fil, alors l'équation (3) peut s'écrire :

$$
\int_{\Gamma} \mathbf{E}(r) \cdot \mathbf{J}^{\prime}(r) \mathrm{d} r=\int_{\Gamma} Z_{\mathrm{c}} \mathbf{J}_{\mathbf{s}}(r) \cdot \mathbf{J}^{\prime}(r) \mathrm{d} r
$$

En remplaçant dans l'équation (9), l'expression de $\mathbf{E}$ donnée par les équations (7) et (8), il vient, après intégration par parties, la formulation variationnelle suivante :

$$
\begin{aligned}
& \int_{\Gamma} \int_{\Gamma} \mathrm{G}\left(\left|r-r^{\prime}\right|\right)\left\{\jmath \omega \mu \mathbf{J}_{\mathrm{s}}\left(r^{\prime}\right) \cdot \mathbf{J}^{\prime}(r)-\frac{j}{\omega \varepsilon}\left(\nabla \cdot \mathbf{J}_{\mathrm{s}}\left(r^{\prime}\right)\right)\left(\nabla \cdot \mathbf{J}_{\mathrm{s}}^{\prime}(r)\right)\right\} \mathrm{d} r \mathrm{~d} r^{\prime}= \\
& -\int_{\Gamma} \mathbf{E}_{i n c}(r) \cdot \mathbf{J}^{\prime}(r) \mathrm{d} r+\int_{\Gamma} \mathrm{Z}_{c} \mathbf{J}_{s}(r) \cdot \mathbf{J}^{\prime}(r) \mathrm{d} r
\end{aligned}
$$

La résolution de cette équation par une méthode d'éléments finis, nous permet d'obtenir le courant $\mathbf{J}_{\mathbf{s}}$ en tout point $\mathbf{d u}$ fil. Les éléments finis utilisés sont de dimension ID et l'objet 
filaire est décrit géométriquement dans les trois dimensions. Le traitement mathématique de l'équation (10) et de son noyau de Green est présenté dans l'appendice A. Disposant de la distribution de courant, il est enfin possible de calculer de façon rigoureuse les champs diffractés dans tout l'espace par l'obstacle filaire.

L'hélice se trouve dans un milieu hôte de caractéristiques radioélectriques $(\varepsilon, \mu)$, et est soumise à une onde électromagnétique. Les champs incidents, par interactions avec l'objet, vont induire des multipôles électriques et magnétiques qui vont rayonner de l'énergie dans toutes les directions. Le champ diffracté par l'hélice, peut se décrire comme la superposition du rayonnement de tous ces multipôles élémentaires. Si l'hélice est de faible dimension devant la longueur d'onde, on simplifie le problème en considérant que parmi tous les multipôles, seuls les moments dipolaires électrique $\mathbf{p}$ et magnétique $\mathbf{m}$, ont une influence significative. Ainsi, dans ce cas, le champ diffracté peut se définir comme la superposition des effets électriques et magnétiques des moments dipolaires de l'hélice. A une distance $r$ de l'objet et dans une direction $\mathbf{n}$, le champ total diffracté par l'inclusion est donné de manière approchée par :

$$
\mathbf{E}_{\mathrm{sc}}=\frac{\beta^{2}}{4 \pi \varepsilon} \frac{\mathrm{e}^{\jmath \beta r}}{r}\left[(\mathbf{n} \times \mathbf{p}) \times \mathbf{n}-\mathbf{n} \times \frac{\mathbf{m}}{c}\right]
$$

Les moments dipolaires $\mathbf{p}$ et $\mathbf{m}$ sont donnés par les formules suivantes :

$$
\begin{aligned}
\mathbf{p} & =\frac{-1}{j \omega} \int \mathbf{J}_{\mathbf{s}}\left(s^{\prime}\right) \mathrm{d} s^{\prime} \\
\mathbf{m} & =\frac{1}{2} \int \mathbf{r} \times \mathbf{J}_{\mathbf{s}}\left(s^{\prime}\right) \mathrm{d} s^{\prime}
\end{aligned}
$$

r représente une distance $O M$ où $O$ est le barycentre géométrique de l'objet filaire et $\mathrm{M}$ un point à la surface du fil ; $\omega$ est la pulsation angulaire de l'onde plane incidente (convention $\exp \{-j \omega t\})$ et $\mathbf{J}_{s}\left(s^{\prime}\right)$ est le courant total induit à la surface de l'objet par l'onde incidente.

Le cas simple de l'hélice modèle (Fig. 1a) permet de comprendre les phénomènes physiques qui interviennent dans ce problème [1]. La composante du champ électrique suivant l'axe de l'hélice induit des courants $i_{E}$ dans les portions droites de l'objet chiral. Ces courants se propagent dans la boucle et créent ainsi un champ magnétique : on peut donc dire que le champ $\mathbf{E}$ contribue dans les portions droites, au moment dipolaire électrique $\mathbf{p}$ et dans la boucle, au moment dipolaire magnétique $\mathbf{m}$ de l'objet chiral. D'autre part, la composante du champ magnétique suivant l'axe de l'hélice induit par la variation de son flux, des courants $i_{H}$ dans la boucle, qui s'écoulent dans les portions droites pour former une accumulation de charges aux extrémités du fil. Ainsi, le champ magnétique incident, participe au moment dipolaire magnétique $\mathbf{m}$ de l'hélice, et par le courant dans les portions linéaires, il contribue également à son moment dipolaire électrique $\mathbf{p}$. Les moments dipolaires de l'objet chiral peuvent alors s'exprimer sous la forme :

$$
\begin{array}{r}
\mathbf{p}=\varepsilon\left(\overline{\bar{\alpha}}_{\mathrm{e}} \mathbf{E}+\overline{\bar{\alpha}}_{\mathrm{em}} \eta \mathbf{H}\right) \\
\mathbf{m}=\frac{\overline{\bar{\alpha}}_{\mathrm{me}}}{\eta} \mathbf{E}+\overline{\bar{\alpha}}_{\mathrm{m}} \mathbf{H}
\end{array}
$$

où $\overline{\bar{\alpha}}_{\mathrm{e}}, \overline{\bar{\alpha}}_{\mathrm{m}}, \overline{\bar{\alpha}}_{\mathrm{em}}$ et $\overline{\bar{\alpha}}_{\mathrm{me}}$ sont des tenseurs, dits tenseurs de polarisabilité et $\eta=\sqrt{\mu / \varepsilon}$ est l'impédance d'onde dans le milieu hôte de caractéristiques radioélectriques $(\varepsilon, \mu)$. Ces relations mettent en évidence un des effets de la chiralité qui est le couplage entre les champs électrique 


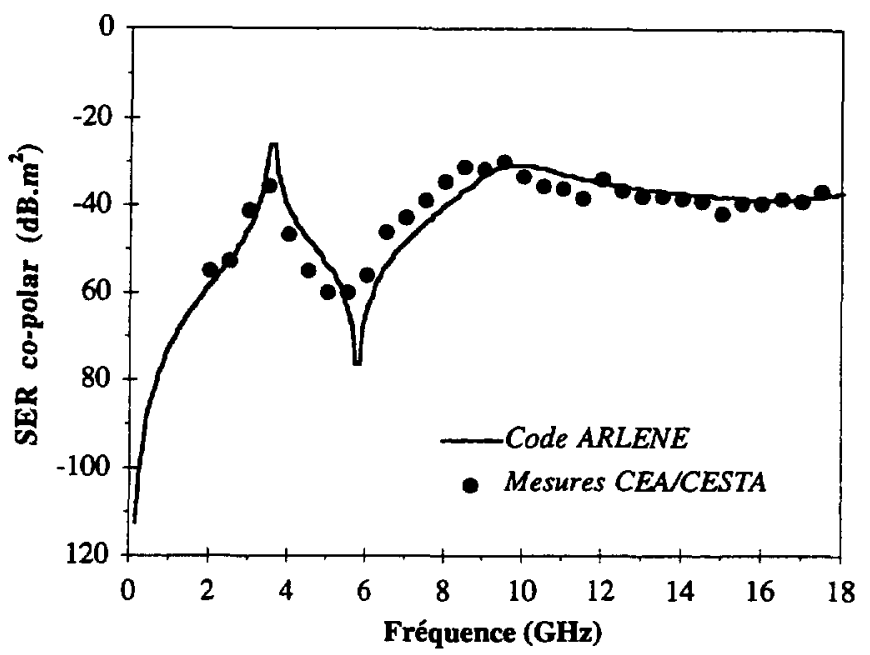

Fig. 3. - SER copolarisée d'une hélice métallique modèle droite (le vecteur d'onde $\mathbf{k}$ est perpendiculaire à l'axe de l'hélice et le champ électrique $\mathbf{E}$ est parallèle à l'axe de l'hélice) : comparaison code ARLENE - mesures en espace libre. Caractéristiques de l'hélice modèle : $R=3 \mathrm{~mm}, L=12 \mathrm{~mm}$, $2 a=0,2 \mathrm{~mm}, \alpha=0,133 \mathrm{rad}, 1$ tour.

[RCS (copolarized component of the scattered field) for a canonical perfectly conducting helix in free-space ( $\mathbf{k}$ perpendicular to helix axis, $\mathbf{E}$ along helix axis): comparison between computations by ARLENE and measurements. Canonical helix dimensions: $R=3 \mathrm{~mm}, L=12 \mathrm{~mm}, a=0.1 \mathrm{~mm}$, $\alpha=0.133$ rad.]

et magnétique créé par la forme chirale de l'objet. Si l'inclusion n'était pas chirale, les polarisabilités "croisées" $\overline{\bar{\alpha}}_{\text {me }}$ et $\overline{\bar{\alpha}}_{\text {em }}$ n'existeraient pas et les moments dipolaires électrique et magnétique seraient alors simplement proportionnels à leurs champs respectifs $\mathbf{E}$ et $\mathbf{H}$. Pour déterminer les composantes de ces tenseurs, on utilise la démarche décrite dans l'appendice B. Cette procédure est similaire, entre autres, à celle de Brewitt-Taylor [17].

En résumé la première partie de ce modèle numérique permet de calculer la densité de courant, les moments dipolaires électrique $(\mathbf{p})$ et magnétique $(\mathbf{m})$ et les tenseurs de polarisabilité électrique $\left(\overline{\bar{\alpha}}_{\mathrm{e}}\right)$, magnétique $\left(\overline{\bar{\alpha}}_{\mathrm{m}}\right)$ et croisées $\left(\overline{\bar{\alpha}}_{\mathrm{me}}\right.$ et $\overline{\bar{\alpha}}_{\mathrm{em}}$ ) pour une hélice isolée ainsi que la diffusion dans toutes les directions de cet objet diffractant, de façon rigoureuse par la formule (7), ou approchée par l'équation (11). Bien entendu cette approche n'est pas restreinte à la seule géométrie des hélices, elle peut s'appliquer à toute inclusion filaire, de géométrie quelconque et de conductivité finie $\left(10^{2}-10^{7} \mathrm{~S} / \mathrm{m}\right)$.

3.1.2. Validations. - Nous avons tout d'abord vérifié la validité des courants et des chamrs diffractés calculés numériquement par le code ARLENE sur un objet chiral isolé. Pour cela sur la figure 3, la SER (Surface Equivalente Radar) copolarisée d'une hélice métallique modèle à un tour, calculée avec le code ARLENE, est comparée à des mesures effectuées en espace libre. On peut apprécier l'accord des résultats obtenus sachant que la précision des mesures de SER est estimée à $\pm 1 \mathrm{~dB} \mathrm{~m}{ }^{2}$. La Surface Equivalente Radar se définit comme le rapport entre champ diffracté et champ incident à grande distance $R$ de l'objet [24].

$$
\operatorname{SER}\left(\mathrm{dB} \mathrm{m} \mathrm{m}^{2}\right)=\lim _{R \rightarrow \infty}\left[10 \log _{10}\left(4 \pi R^{2} \frac{\mathbf{E}_{\mathrm{d}} \cdot \mathbf{E}_{\mathrm{d}}^{*}}{\mathbf{E}_{\mathrm{inc}} \mathbf{E}_{\mathrm{inc}}^{*}}\right)\right]
$$



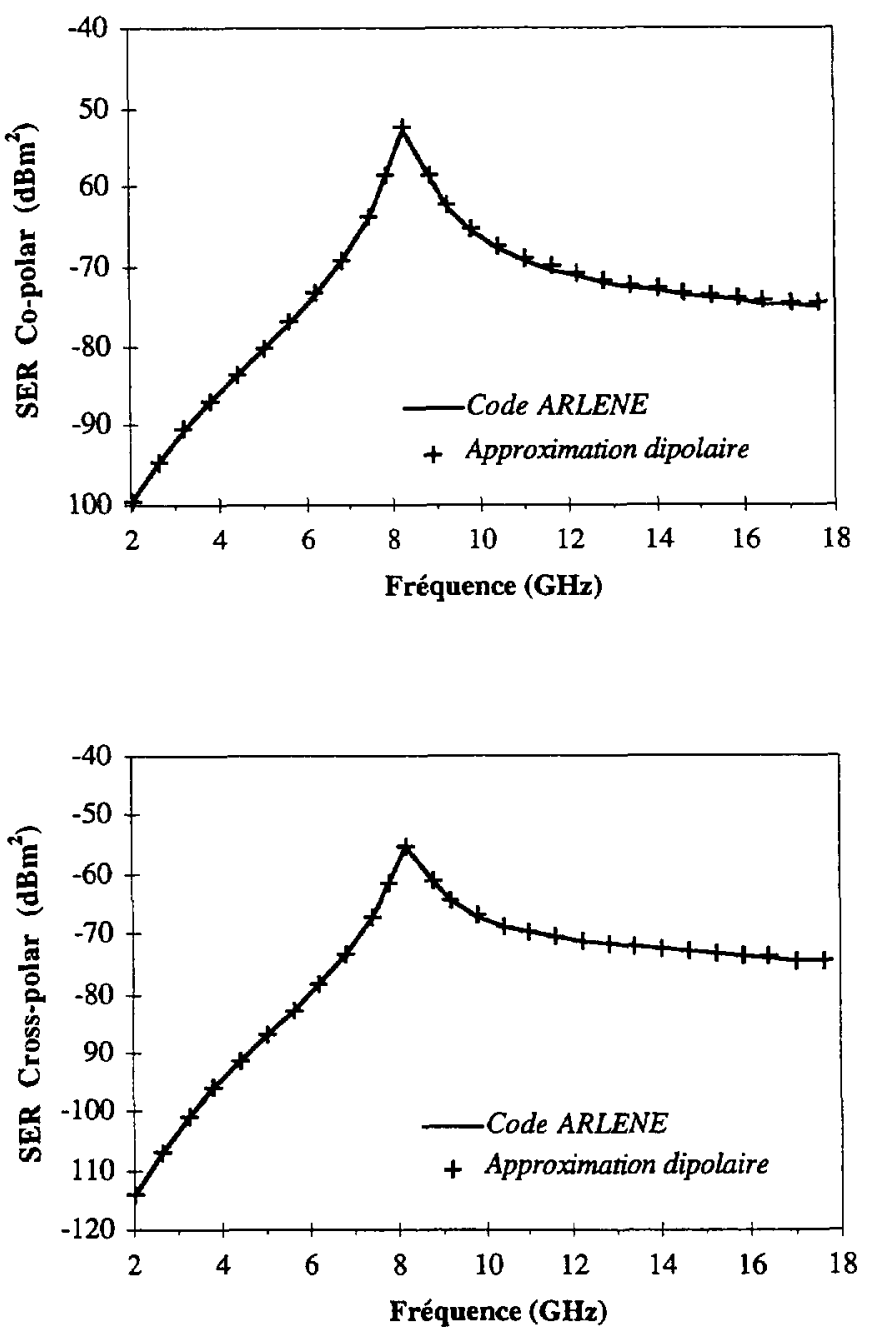

Fig. 4. - Validation de l'approximation dipolaire pour simuler un petit objet diffractant (le vecteur d'onde $\mathbf{k}$ est perpendiculaire à l'axe de l'hélice et le champ magnétique $\mathbf{H}$ est parallèle à l'axe de l'hélice). Caractéristiques de l'objet diffractant (hélice) : $R=1 \mathrm{~mm}$, Pas=1 $\mathrm{mm}, 2 a=0,2 \mathrm{~mm}, 2$ tours à droite, $\varepsilon=\varepsilon_{0}(1,8+\jmath 0,1)$ et $\mu=\mu_{0}$.

[Validation of the dipole approximation for small bi-anisotropic scatterer ( $\mathbf{k}$ perpendicular to helix axis, $\mathbf{H}$ along helix axis). Helix dimensions: $R=1 \mathrm{~mm}, \mathrm{Pitch}=1 \mathrm{~mm}, 2 a=0.2 \mathrm{~mm}, 2$ turns and left-handed. Host medium characteristics $\varepsilon=\varepsilon_{0}(1.8+j 0.1)$ and $\mu=\mu_{0}$.]

Le symbole $\left(^{*}\right)$ représente le nombre complexe conjugué. La SER copolarisée s'obtient en considérant seulement la composante du champ diffracté qui a la même direction que le champ électrique incident. La SER crosspolarisée se calcule de manière analogue, à partir du champ diffracté dans la direction orthogonale au champ électrique incident.

Nous avons ensuite vérifié que l'approximation dipolaire (Eq. (11)) que nous avons utilisée au paragraphe précédent est justifiée. Pour cela, nous avons comparé la SER donnée par le code ARLENE aux résultats obtenus par la formule approchée (Eq. (11)). Sur la figure 4, les 

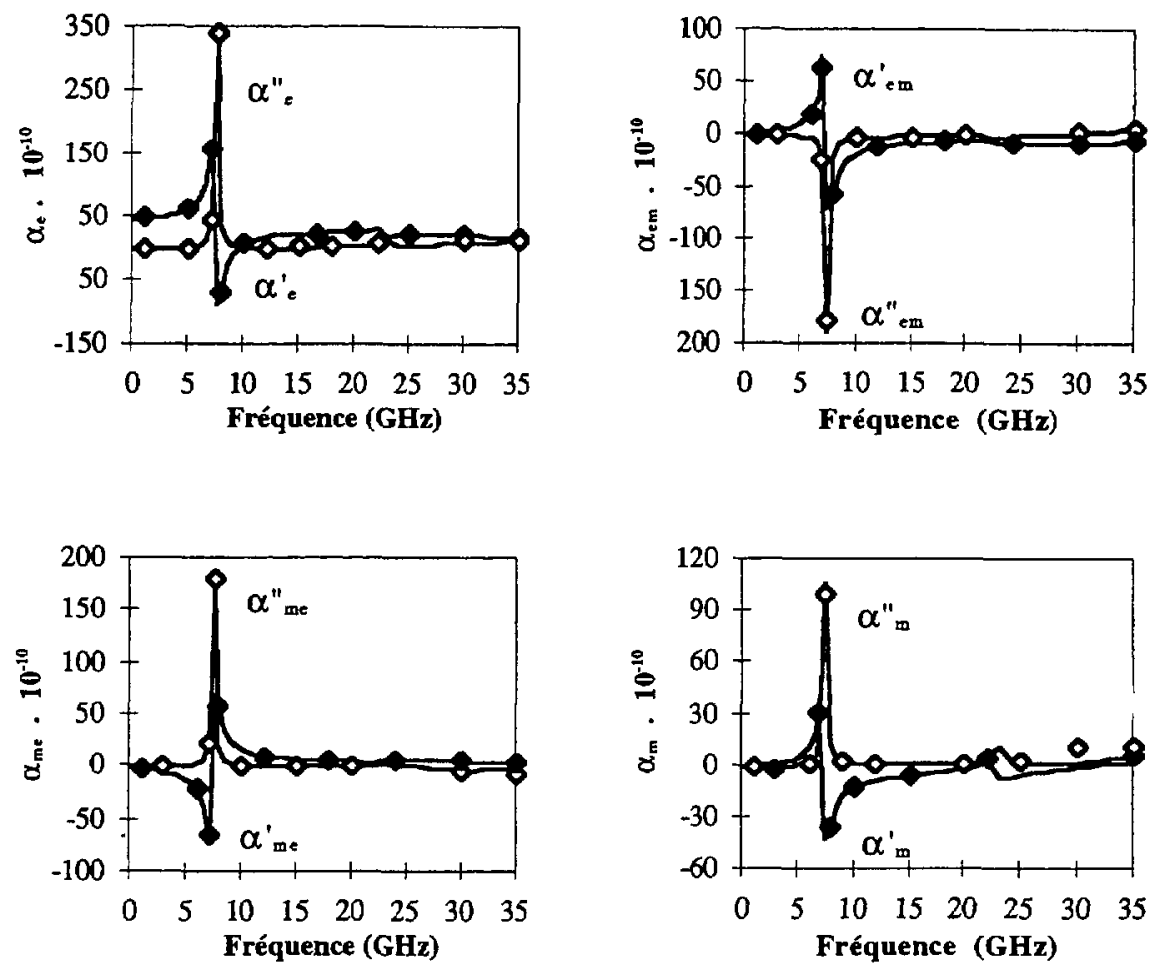

Fig. 5. - Polarisabilités moyennes d'une hélice métallique à 3 tours (pas à gauche) parfaitement conductrice noyée dans un liant de caractéristiques $\varepsilon=\varepsilon_{0}(3,2+j 0,128)$ et $\mu=\mu_{0}$. Caractéristiques de l'hélice : $R=0,5842 \mathrm{~mm}$, Pas $=0,529 \mathrm{~mm}, 2 a=0,1524 \mathrm{~mm}$.

[Scalar polarizabilities for a 3-turns perfectly conducting helix in host material characteristics used in the computations: $\varepsilon=\varepsilon_{0}(3.2+j 0.128)$ and $\mu=\mu_{0}$. Helix dimensions: $R=0.5842 \mathrm{~mm}$, Pitch $=0.529 \mathrm{~mm}, 2 a=0.1524 \mathrm{~mm}$.]

calculs ont été menés pour une hélice à 2 tours et pas à droite. On peut observer un très bon accord entre les deux courbes, et ce, même au-delà de la première fréquence de résonance. Ainsi, dans les bandes de fréquences auxquelles nous nous sommes intéressés, il semble satisfaisant de représenter une hélice par ses moments dipolaires de premier ordre $\mathbf{p}$ et $\mathbf{m}$.

Enfin sur la figure 5, nous validons le calcul des tenseurs de polarisabilité en comparant les résultats obtenus par notre modèle avec ceux donnés par une modélisation propre à Thomson CSF [15]. Ils utilisent l'amplitude moyenne de diffusion d'une hélice vers l'avant et vers l'arrière, pour calculer ensuite, les 2 nombres d'ondes (circulaire droit et circulaire gauche) ainsi que l'impédance d'onde. Ces grandeurs permettent de caractériser la propagation dans le matériau chiral et de remonter ensuite aux paramètres effectifs du composite. Conıme il est difficile de comparer tous les termes de ces tenseurs, nous présentons sur la figure 5 des valeurs scalaires, définies comme la moyenne des termes diagonaux. Ces valeurs moyennes ont été choisies car elles ont un sens physique et entrent explicitement dans la modélisation des propriétés effectives des composites à inclusions chirales réparties de façon aléatoire dans un milieu hôte. Les hélices étant des objets réciproques, on vérifie bien que les valeurs moyennes $\alpha_{\text {me }}$ et $\alpha_{\text {em }}$ des tenseurs de polarisabilité $\overline{\bar{\alpha}}_{\text {me }}$ et $\overline{\bar{\alpha}}_{\text {em }}$ sont égales au signe près, on pose alors : $\alpha_{\mathrm{em}}=-\alpha_{\mathrm{me}}=\alpha_{\mathrm{c}}=$ $\left(\alpha_{\mathrm{em} 11}+\alpha_{\mathrm{em} 22}+\alpha_{\mathrm{em} 33}\right) / 3$. On peut apprécier sur la figure 5 le bon accord entre les deux modèles. 
3.1.3. Discussion. - Sur les figures 3, 4 et 5, la Surface Equivalente Radar et les différentes polarisabilités moyennes d'une hélice présentent un caractère résonnant en fonction de la fréquence. La valeur de cette fréquence de résonance se retrouve par la loi empirique suggérée par exemple dans les références $[13,17]: f_{\text {res }} \approx c /(2 n L)$, où $c$ est la vitesse des ondes électromagnétiques dans le vide, $n$ la partie réelle de l'indice de réfraction du milieu hôte et $L$ la longueur étirée de l'hélice. En fait, malgré sa forme enroulée, l'hélice se comporte principalement comme une antenne dipôle filaire et résonne en $\lambda / 2$.

\subsection{CALCUL DES PROPRIÉtÉS EFFECTIVES DU COMPOSITE}

3.2.1. Principe. - Connaissant les propriétés électromagnétiques d'une hélice isolée noyée dans un milieu hôte quelconque avec ou sans pertes, il s'agit ensuite de déterminer les propriétés effectives $\left(\varepsilon_{\mathrm{c}}, \mu_{\mathrm{c}}\right.$ et $\left.\chi_{\mathrm{c}}\right)$ du matériau composite. Pour cela on suppose qu'une hélice peut être représentée par ses moments dipolaires électrique et magnétique sur une large bande de fréquences (propriété vérifiée au paragraphe 3.1.2), puis on emploie une loi de mélange de type Maxwell Garnett, qui permet de tenir compte de l'interaction au premier ordre entre des particules distribuées de manière aléatoire.

D'un point de vue macroscopique, les propriétés électromagnétiques d'un milieu isotrope peuvent être décrites à l'aide des vecteurs polarisation $\mathbf{P}$ et aimantation $\mathbf{M}$ :

$$
\begin{aligned}
& \mathbf{D}=\varepsilon \mathbf{E}+\mathbf{P} \\
& \mathbf{B}=\mu(\mathbf{H}+\mathbf{M})
\end{aligned}
$$

où $\varepsilon$ et $\mu$ sont les permittivité et perméabilité complexes du milieu hôte entourant les hélices. Les vecteurs polarisation $\mathbf{P}$ et aimantation $\mathbf{M}$ sont respectivement la somme de tous les moments dipolaires électriques et magnétiques du milieu par unité de volume. Pour un composite chiral isotrope, où les inclusions sont réparties de façon aléatoire dans le liant, les vecteurs polarisation $\mathbf{P}$ et aimantation $\mathbf{M}$ s'expriment sous la forme [9] :

$$
\begin{aligned}
\mathbf{P} & =N \mathbf{p}=N \varepsilon\left(\alpha_{\mathrm{e}} \mathbf{E}_{\mathrm{loc}}+\alpha_{\mathrm{c}} \eta \mathbf{H}_{\mathrm{loc}}\right) \\
\mathbf{M} & =N \mathbf{m}=N\left(\frac{\alpha_{\mathrm{c}}}{\eta} \mathbf{E}_{\mathrm{loc}}+\alpha_{\mathrm{m}} \mathbf{H}_{\mathrm{loc}}\right)
\end{aligned}
$$

où $\alpha_{\mathrm{e}}, \alpha_{\mathrm{m}}$ et $\alpha_{\mathrm{c}}$ sont les polarisabilités scalaires (moyenne des termes diagonaux des tenseurs $\overline{\bar{\alpha}}_{\mathrm{e}}, \overline{\bar{\alpha}}_{\mathrm{m}}, \overline{\bar{\alpha}}_{\text {em }}$ et $\overline{\bar{\alpha}}_{\text {me }}$ qui caractérisent une inclusion chirale) et $N$ le nombre d'objets chiraux par unité de volume introduits dans le matériau. On remarquera dans ces formules que les vecteurs $\mathbf{E}$ et $\mathbf{H}$ ont été remplacés par $\mathbf{E}_{\text {loc }}$ et $\mathbf{H}_{\text {loc }}$ qui sont les champs locaux au niveau de la particule chirale. On considère en fait qu'ils représentent le champ moyen incident auquel on ajoute la contribution des particules environnantes. En considérant des interactions purement Lorentziennes et en s'inspirant de la géométrie sphérique, les champs locaux s'écrivent :

$$
\begin{aligned}
& \mathbf{E}_{\text {loc }}=\mathbf{E}+\mathbf{P} / 3 \varepsilon \\
& \mathbf{H}_{\text {loc }}=\mathbf{H}+\mathbf{M} / 3
\end{aligned}
$$

En combinant les équations (19) et (20) avec les formules (21) et (22), on exprime $\mathbf{P}$ et $\mathbf{M}$ en fonction des champs $\mathbf{E}$ et $\mathbf{H}$. On reporte ces résultats dans les formules (17) et (18), puis, 
en identifiant avec les équations constitutives (1) et (2), on obtient les paramètres effectifs du matériau sous la forme :

$$
\begin{aligned}
\varepsilon_{\mathrm{c}} & =\varepsilon \frac{\left(1+2 N \alpha_{\mathrm{e}} / 3\right)\left(1-N \alpha_{\mathrm{m}} / 3\right)-2 N^{2} \alpha_{\mathrm{c}}^{2} / 9}{\left(1-N \alpha_{\mathrm{e}} / 3\right)\left(1-N \alpha_{\mathrm{m}} / 3\right)+N^{2} \alpha_{\mathrm{c}}^{2} / 9} \\
\mu_{\mathrm{c}} & =\mu \frac{\left(1+2 N \alpha_{\mathrm{m}} / 3\right)\left(1-N \alpha_{\mathrm{e}} / 3\right)-2 N^{2} \alpha_{\mathrm{c}}^{2} / 9}{\left(1-N \alpha_{\mathrm{e}} / 3\right)\left(1-N \alpha_{\mathrm{m}} / 3\right)+N^{2} \alpha_{\mathrm{c}}^{2} / 9} \\
j \chi_{\mathrm{c}} & =\frac{-N \alpha_{\mathrm{c}} \sqrt{\varepsilon \mu}}{\left(1-N \alpha_{\mathrm{e}} / 3\right)\left(1-N \alpha_{\mathrm{m}} / 3\right)+N^{2} \alpha_{\mathrm{c}}^{2} / 9}
\end{aligned}
$$

Les équations (23) sont équivalentes à celles obtenues par Sihvola et al. [9]. Connaissant ces paramètres, il est ensuite possible d'étudier les propriétés de réflexion, de transmission et bien sûr de réflexion sur court-circuit d'une couche ou d'un multicouche de matériau chiral. Le modèle numérique que nous avons développé et qui permet de calculer les propriétés effectives d'un composite à inclusions métalliques filaires (chirales ou non) a été baptisé le modèle MTWC (Modelling of Thin Wire Composites).

3.2.2. Validations. - Les calculs de l'interaction d'une onde électromagnétique avec une hélice étant validés, étudions les résultats du modèle MTWC qui permet d'obtenir le comportement radioélectrique d'un composite chiral. Les valeurs expérimentales (mesures en espace libre) et théoriques (modèle MTWC) de la permittivité effective d'un composite chiral, de l'angle de rotation et de l'ellipticité, des coefficients de transmission (co- et cross- polarisation) et de réflexion (devant court-circuit) d'une couche chirale sont présentés sur les figures 6 à 9 . Les mesures des figures 6 et 7 ont été effectuées à l'IRCOM de Limoges. Elles sont réalisées sur un banc focalisé en espace libre. La bande 6-18 GHz est obtenue à l'aide de 3 paires de cornets avec lentilles diélectriques $(5,6-8,2 \mathrm{GHz}$ puis $8,2-12,4 \mathrm{GHz}$ et enfin $12,4-18 \mathrm{GHz})$. Pour les figures 8 et 9 , les mesures ont été effectuées en champ lointain dans les chambres anéchoïques du CEA/CESTA. Les mesures de réflectivité en incidence normale sont réalisées en mode monostatique et l'illumination du matériau est assurée par une batterie de 6 cornets couvrant la bande 1,7-18 GHz. Pour les coefficients de transmission, 2 cornets à double polarisation couvrant la bande $2-18 \mathrm{GHz}$ sont utilisés.

A travers ces différents exemples, on peut remarquer que les résultats de la modélisation reproduisent l'allure générale des différents types de mesures effectuées sur des composites chiraux et en particulier au voisinage des zones de résonance. La mesure de l'angle de rotation et de l'ellipticité permet de valider le calcul du coefficient de chiralité. En effet, dans le formalisme employé (Eqs. (1) et (2)) ces grandeurs sont reliées simplement, respectivement à la partie réelle et à la partie imaginaire de $\chi_{\mathrm{c}}$ (pour une couche d'épaisseur $e$ ) :

$$
\begin{aligned}
& \text { Rotation }\left(^{\circ}\right)=-\omega \chi_{\mathrm{c}}^{\prime} e \frac{180}{\pi} \\
& \text { Ellipticité }=-\omega \chi_{\mathrm{c}}^{\prime \prime} e
\end{aligned}
$$

On note cependant sur la figure 9, que la modélisation présente un écart important avec les mesures à partir de $14 \mathrm{GHz}$. En effet, dans ce cas on se trouve dans les limites de l'approximation filaire. En général les méthodes intégrales imposent une discrétisation de l'objet en $\lambda / 10$. Dans ces conditions, pour $18 \mathrm{GHz}$, la longueur du segment filaire doit rester inférieure à $0,93 \mathrm{~mm}$. Le diamètre du fil étant de $0,95 \mathrm{~mm}$, l'objet étudié est un cylindre et non plus un fil "infiniment mince", la cellule élémentaire considérée ne vérifie donc plus les conditions d'approximation 


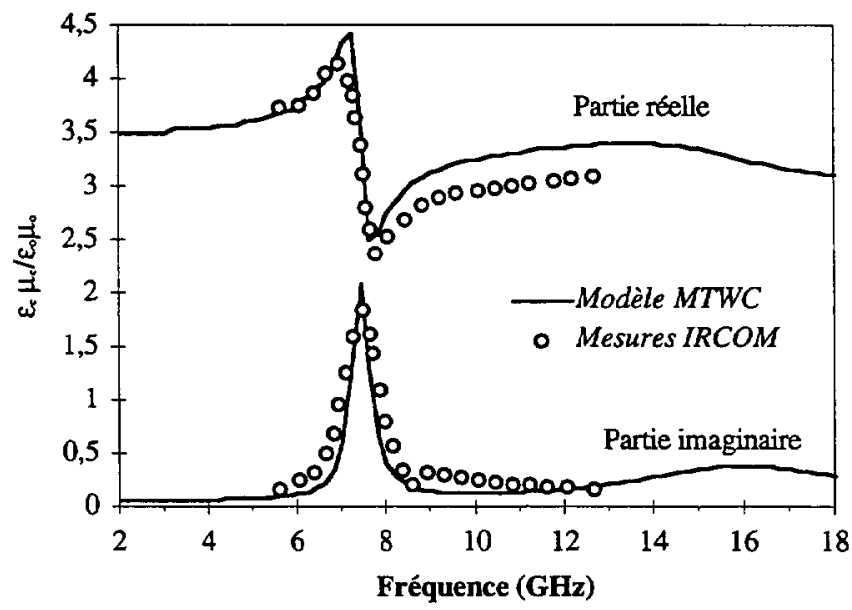

Fig. 6. - Permittivité effective (produit $\varepsilon_{\mathrm{c}} \mu_{\mathrm{c}} / \varepsilon_{0} \mu_{0}$, pour la mesure on a supposé $\mu_{\mathbf{c}}=\mu_{0}$ ) d'un milieu chiral en fonction de la fréquence : comparaison mesures - modèle MTWC. Le composite chiral à base d'hélices métalliques parfaitement conductrices à 1 tour gauche a été fabriqué par le CEA/Saclay [25, 26]. Caractéristiques des hélices : $R=1,65 \mathrm{~mm}$, Pas $=2 \mathrm{~mm}, a=0,15 \mathrm{~mm}$ et $N=3,1$ hélices $/ \mathrm{cm}^{3}$. Caractéristiques du liant : $\varepsilon=\varepsilon_{0}(3,2+j 0,066)$ et $\mu=\mu_{0}$.

[Relative complex permittivity for a chiral composite versus frequency (product $\varepsilon_{\mathrm{c}} \mu_{\mathrm{c}} / \varepsilon_{0} \mu_{0}$, during measurement $\mu_{\mathrm{c}}=\mu_{0}$ was supposed): comparison between computations and measurements. Chiral composite with randomly oriented regular perfectly conducting helices was manufactured by CEA/Saclay [25, 26]. Helix dimensions: $R=1.65 \mathrm{~mm}$, Pitch $=2 \mathrm{~mm}, a=0.15 \mathrm{~mm}, 1$ turn and left-handed. Helix density: 3.1 helices $/ \mathrm{cm}^{3}$. Host material characteristics: $\varepsilon=\varepsilon_{0}(3.2+j 0.066)$ and $\mu=\mu_{0}$.]

filaire. Néanmoins, en dehors de cette zone, les valeurs du coefficient de réflexion sont en bon accord avec les résultats expérimentaux.

On peut donc dire que MTWC modélise de façon satisfaisante les matériaux hétérogènes chiraux à faibles ou moyennes concentrations, composés d'hélices "modèles" (Fig. 8) ou d'hélices à $n$-tours (Figs. 6 et 7), qu'elles soient parfaitement conductrices ou de conductivité finie (Fig. 9).

3.2.3. Limitations. - Pour obtenir les propriétés effectives, nous utilisons la loi de mélange de type Maxwell Garnett. Cette technique a été développée initialement pour l'étude des composites à inclusions diélectriques. Dans ce domaine les limitations du modèle sont connues et l'on estime en général que la concentration ne doit pas dépasser $10 \%$ en volume.

Pour les objets chiraux, nous utilisons une version modifiée de cette loi de mélange [9]. Si l'on veut définir un pourcentage d'hétérogénéité comme pour les composites diélectriques, il faut utiliser le taux de métal introduit dans le matériau. Dans tous les exemples précédents, ce volume de métal ne dépasse pas $0,3 \%$ (sauf dans le cas particulier de la figure 9 où il atteint $5 \%$ ). Toutefois, d'un point de vue technologique, cette grandeur n'est pas très représentative compte tenu de la forme plutôt complexe des objets chiraux et l'on préférera définir le taux de remplissage comme la fraction de volume occupée par les inclusions :

$$
T(\%)=100 \text { ( } N \text { hélices/unité de volume) (Volume occupé équivalent) }
$$

Le problème réside alors dans le calcul du volume occupé équivalent. Si l'on considère que 

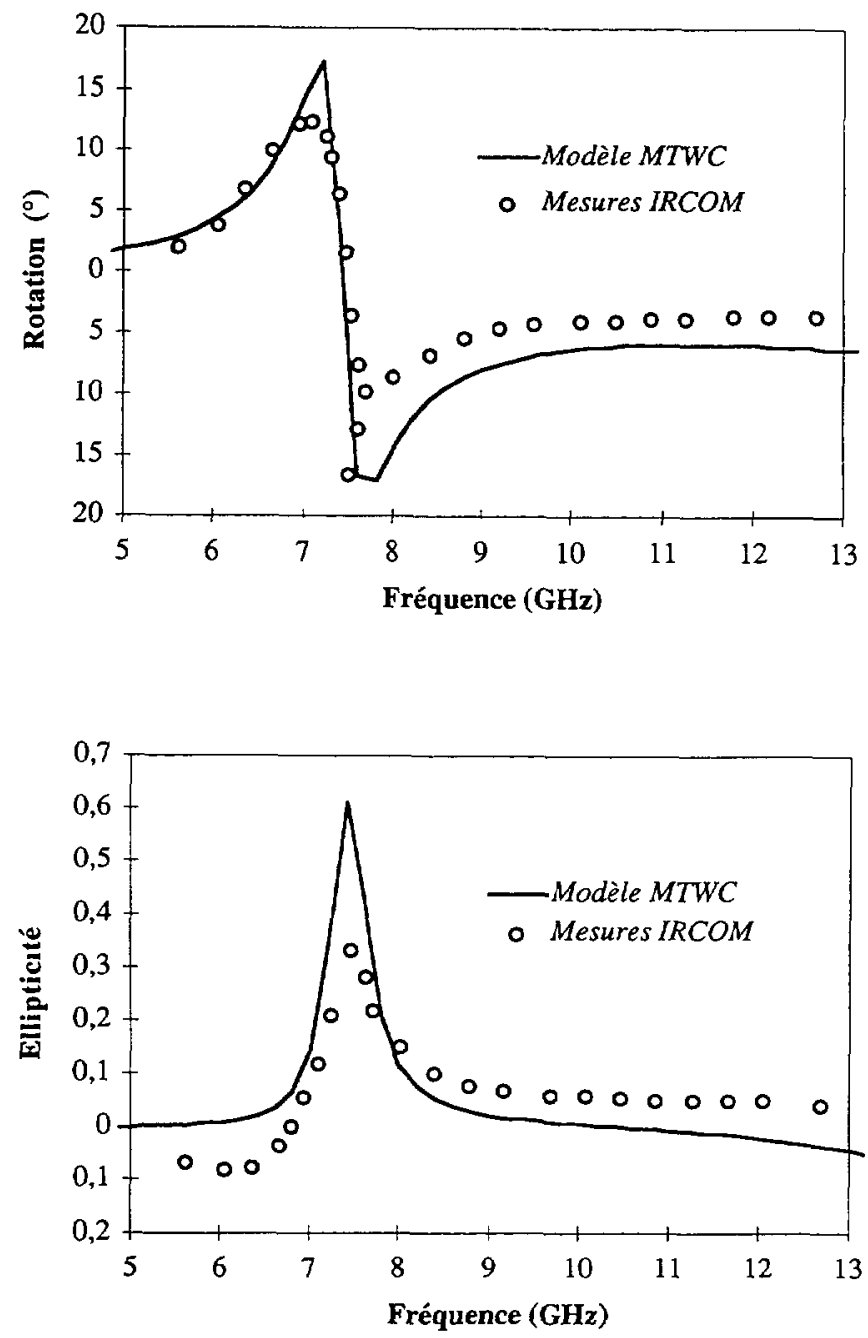

Fig. 7. - Rotation et ellipticité d'une couche chirale en fonction de la fréquence : comparaison mesures - modèle MTWC (composite de même composition que celui de la Fig. 6). Epaisseur de la couche chirale : $15 \mathrm{~mm}$.

[Rotation angle and ellipticity of a chiral slab with randomly oriented regular perfectly conducting helices (same as in Fig. 6): comparison between computations and measurements. Slab thickness: $15 \mathrm{~mm}$.]

l'encombrement spatial d'une hélice correspond à un cylindre, on obtient le volume de remplissage cylindrique $\left(T_{\text {cyl }}\right)$. Si par contre, le volume de l'inclusion est assimilé à la plus petite sphère contenant l'hélice, on définit alors le volume de remplissage sphérique $\left(T_{\mathrm{sph}}\right)$. Ce dernier est bien sur toujours plus important que le précédent.

Nous avons vu précédemment que pour des matériaux relativement peu chargés, le modèle donnait de bons résultats (cas des Figs. 6 et $7: T_{\text {cyl }}=7,26 \% T_{\text {sph }}=8,76 \%$; cas de la Fig. 8 : $T_{\mathrm{cyl}}=10 \% T_{\mathrm{sph}}=15,2 \%$ ). Pour tenter de cerner les limitations de la loi de mélange dans 

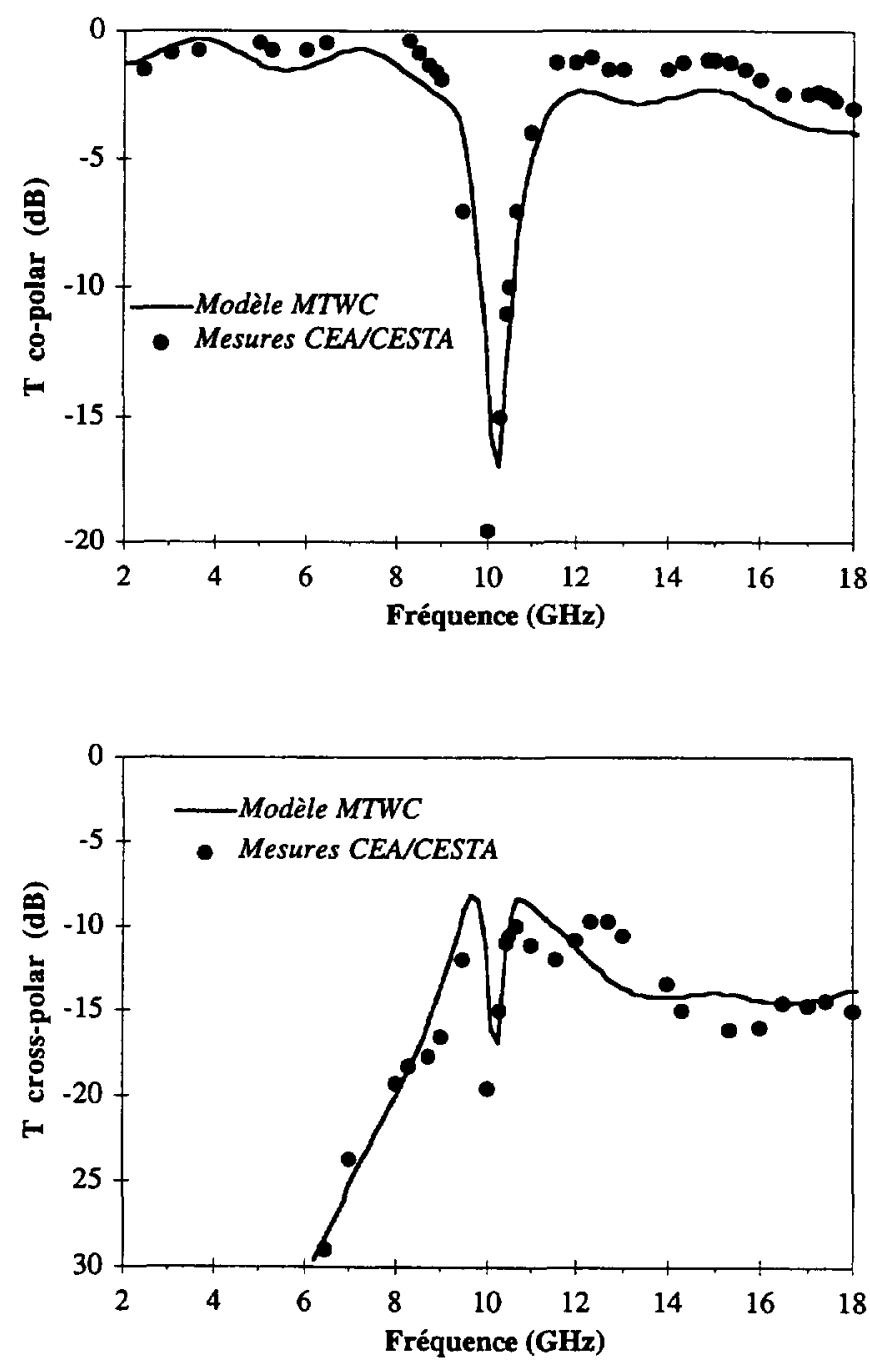

Fig. 8. - Coefficient de transmission (co- et cross-polarisation) d'une couche chirale en fonction de la fréquence : comparaison mesures - modèle MTWC. Le composite chiral à base d'hélices "modèles" métalliques parfaitement conductrices a été fabriqué par le CEA/CER [27]. Caractéristiques des hélices : $R=1,1 \mathrm{~mm}, L=1 \mathrm{~mm}, a=0,1 \mathrm{~mm}, a=0,4$ rad et $N=10$ hélices $/ \mathrm{cm}^{3}$. Caractéristiques du liant : $\varepsilon=\varepsilon_{0}(2,6+\jmath 0,05)$ et $\mu=\mu_{0}$. Epaisseur de la couche : $39 \mathrm{~mm}$.

[Copolarized and crosspolarized transmission coefficients of a chiral slab: comparison between computations and measurements. Chiral composite with randomly oriented canonical perfectly conducting helices was manufactured by CEA/CER [27]. Canonical helix dimensions: $R=1.1 \mathrm{~mm}$, $L=1 \mathrm{~mm}, a=0.1 \mathrm{~mm}, \alpha=0.4 \mathrm{rad}$. Helix density: 10 helices $/ \mathrm{cm}^{3}$. Host material characteristics: $\varepsilon=\varepsilon_{0}(2.6+\jmath 0.05)$ and $\mu=\mu_{0}$. Slab thickness: $39 \mathrm{~mm}$.]

le cadre de cette modélisation, nous avons réalisé l'expérience suivante. Nous avons d'abord effectué la simulation d'un composite contenant 27 hélices $/ \mathrm{cm}^{3}$. Ensuite, nous avons modélisé un composite contenant 13,5 inclusions $/ \mathrm{cm}^{3}$, mais dans ce cas l'inclusion considérée (Fig. 10) 


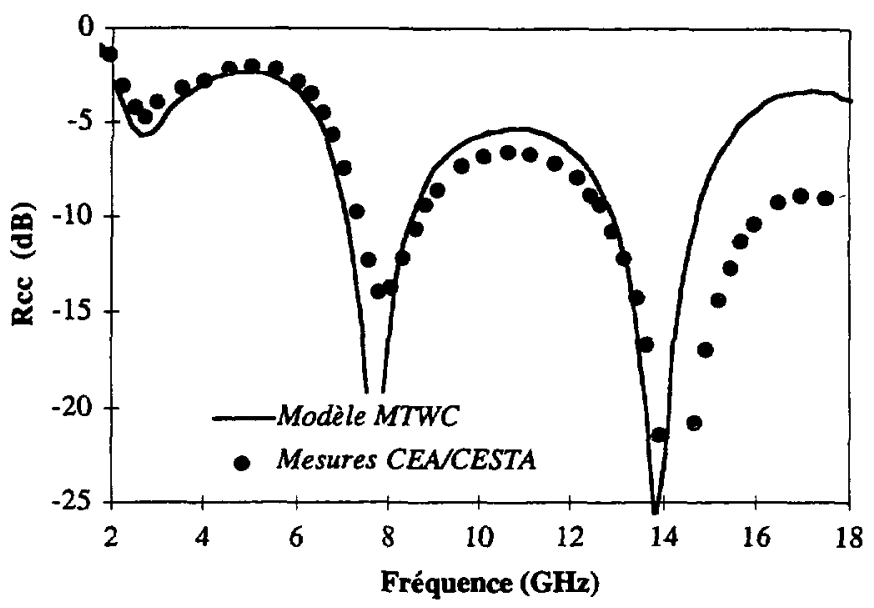

Fig. 9. - Coefficient de réflexion (devant court circuit) d'une couche chirale en fonction de la fréquence: comparaison mesures - modèle MTWC. Le composite chiral à base d'hélices à deux tours en polymère conducteur a été fabriqué par le CEA/Saclay $[25,26]$. Caractéristiques des hélices : $R=1,875 \mathrm{~mm}$, Pas $=2 \mathrm{~mm}, a=0,475 \mathrm{~mm}, 2$ tours gauche et $N=3,1$ hélices $/ \mathrm{cm}^{3}$. Conductivité du polymère conducteur : $\sigma=100 \mathrm{~S} / \mathrm{m}$. Caractéristiques du liant $: \varepsilon=\varepsilon_{0}(3,2+j 0,066)$ et $\mu=\mu_{0}$. Epaisseur de la couche chirale : $15 \mathrm{~mm}$.

[Absolute value of the reflection coefficient from a metal-backed lossy chiral slab as a function of frequency: comparison between computations and measurements. The chiral composite with randomly oriented regular no perfectly conducting helices (intrinsically conductive polymer) was manufactured by CEA/Saclay [25, 26]. Helix dimensions: $R=1.875 \mathrm{~mm}$, Pitch $=2 \mathrm{~mm}, a=0.475 \mathrm{~mm}, 2$ turns and left-handed. Helix density: 3.1 helices $/ \mathrm{cm}^{3}$. Conductivity of the conductive polymer: $\sigma=100 \mathrm{~S} / \mathrm{m}$. Host material characteristics: $\varepsilon=\varepsilon_{0}(3.2+j 0.066)$ and $\mu=\mu_{0}$. Slab thickness: $15 \mathrm{~mm}$.]

est constituée de deux hélices séparées par une distance $d$ (qui a été calculée comme la distance moyenne entre deux inclusions lors de la première simulation). On a réalisé ainsi deux simulations du même composite. Il existe cependant une différence sensible entre les deux calculs puisque dans le deuxième cas, les courants obtenus, tiennent compte du couplage électromagnétique qui existe entre les deux hélices voisines. Ce couplage va donc se répercuter dans le calcul des moments dipolaires, puis dans celui des polarisabilités et enfin dans les paramètres effectifs du composite. Bien sûr le couplage entre les deux hélices étant lié à l'orientation relative entre les deux objets, nous avons placé le deuxième ressort dans trois positions différentes. La figure 11 présente les paramètres effectifs obtenus pour les différentes simulations. Même si les valeurs ne sont pas strictement identiques, les résultats restent voisins. Le couplage électromagnétique entre deux inclusions n'est donc pas assez fort pour influencer radicalement les grandeurs macroscopiques du composite. Ce résultat est assez remarquable surtout si l'on considère que les taux de remplissage sont élevés: $T_{\text {cyl }}=22,6 \%, T_{\mathrm{sph}}=39,3 \%$ et $T_{\text {métal }}=1,608 \%$.

Il est donc délicat d'exprimer la limite de validité de la loi de mélange de Maxwell Garnett appliquée aux matériaux chiraux. En particulier il est difficile de définir une concentration limite comme dans le cas des composites diélectriques. Toutefois il semble que, pour des taux de remplissage cylindrique d'environ 15-20\%, on obtienne encore des résultats corrects.

3.3. Conclusions. - Nous avons pu voir dans ce chapitre, que deux étapes interviennent dans la modélisation des matériaux chiraux de type "milieux effectifs": une première étape 


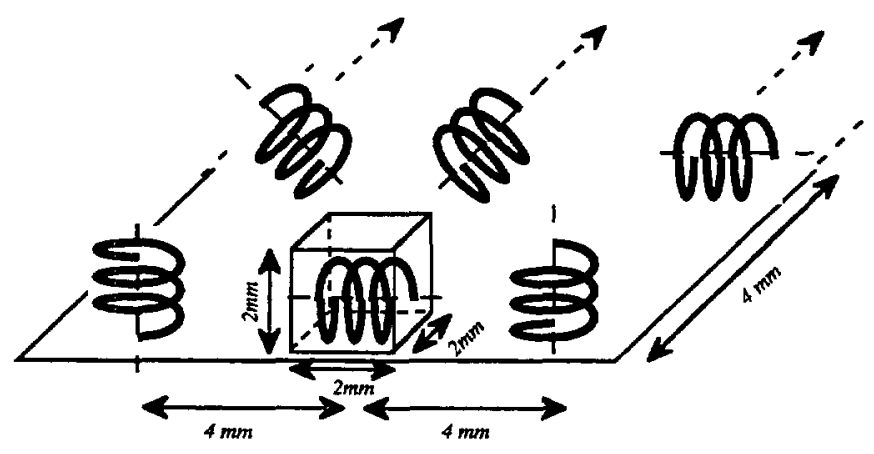

a)

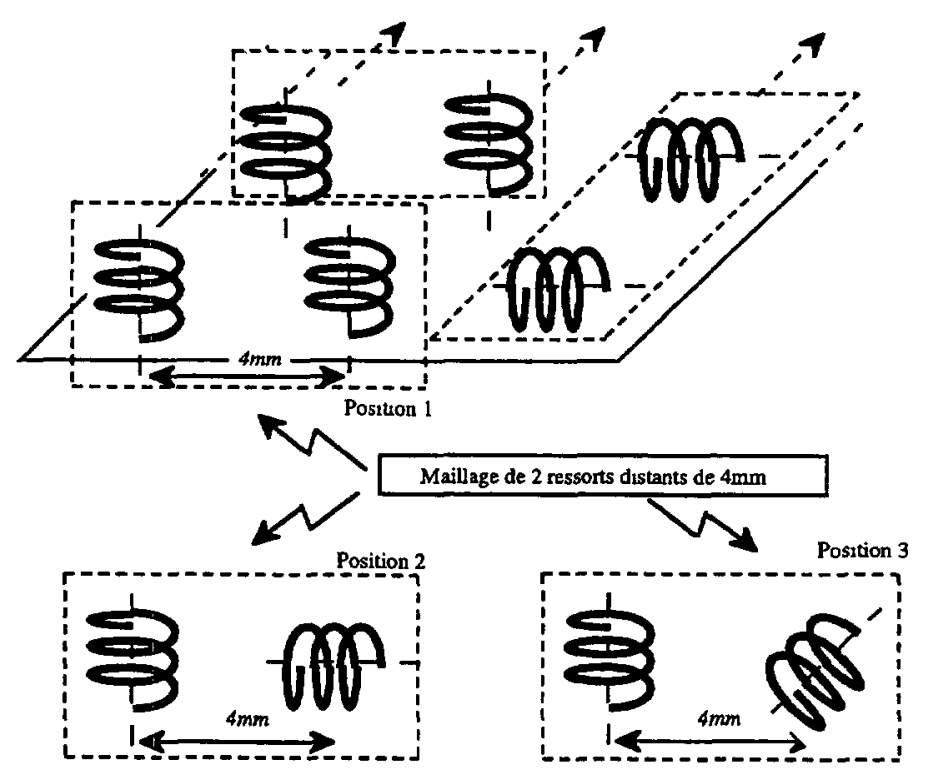

b)

Fig. 10. - a) Exemple de répartition aléatoire des objets chiraux dans les trois dimensions. b) Simulation de l'interaction entre deux hélices voisines pour différentes orientations relatives.

(a) Isotropic distribution of chiral objects in the host medium. b) Coupling simulation between two adjacent helices for different positions.]

qui caractérise la diffraction d'un objet chiral isolé, et une deuxième qui évalue les propriétés effectives du composite par une loi de mélange de type Maxwell Garnett. Chacune de ces deux étapes a été validée et on note un bon accord général entre les mesures et l'approche théorique. Le modèle numérique MTWC permet donc de calculer les propriétés effectives de composites à inclusions chirales (ou non) de formes quelconques (hélices à $n$ tours, oméga, motifs chiraux plans, fibres, boucles...).

En toute rigueur, ce modèle ne s'applique qu'à des composites chiraux à faibles ou moyennes concentrations. Il nous faudrait étudier les interactions "courtes" entre hélices pour pouvoir 

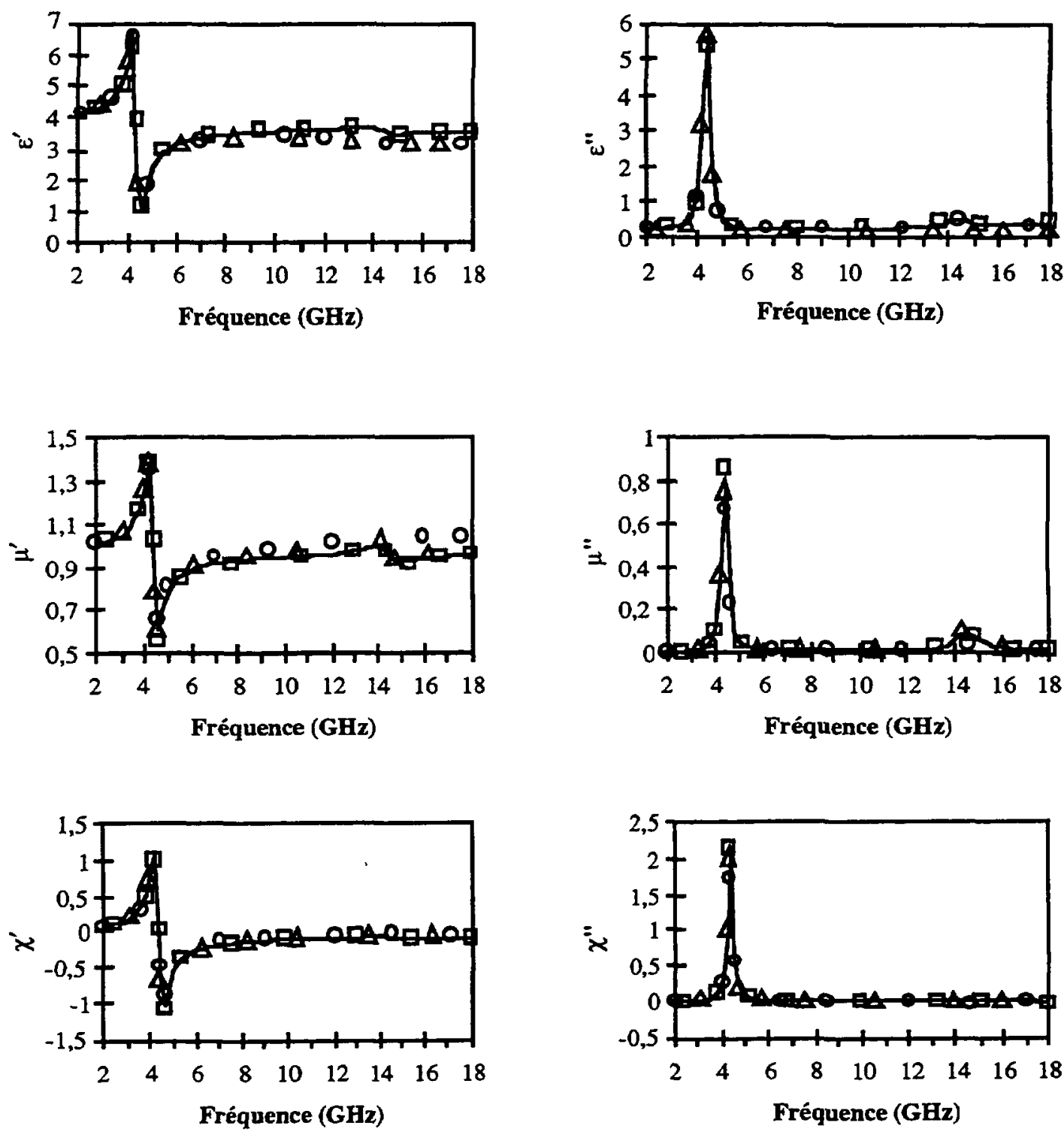

$\longrightarrow \mathrm{N}=27$ hélices $/ \mathrm{cm}^{3}$

口ロ $\mathrm{N}=13,5$ hélices $/ \mathrm{cm}^{3}$ - Position 1

$\Delta \Delta \Delta \quad \mathrm{N}=13,5$ hélices $/ \mathrm{cm}^{3}$ - Position 2

○० $\mathrm{N}=13,5$ hélices $/ \mathrm{cm}^{3}$ - Position 3

Fig. 11. - Simulation de l'interaction entre deux hélices. Comparaison des paramètres effectifs.

[Coupling simulation between two helices. Effective parameters comparison.] 
modéliser les composites chiraux à plus fortes concentrations : pour de tels matériaux, la modélisation risque de se révéler très lourde (modèles de multidiffusion ?). Il est à noter toutefois que l'utilisation de composites chiraux à trop fortes densités risque de poser des problèmes technologiques de mise en œuvre du matériau : enchevêtrement des hélices, percolation .

\section{Matériaux chiraux et absorbants micro-ondes}

En ce qui concerne les absorbants micro-ondes, dans un premier temps nous avons calculé théoriquement leurs performances en tant qu'absorbants monocouches, bicouches ou multicouches (coefficient de réflexion, réponse angulaire, réponse en fréquence), en fonction du nombre de couches, de leurs épaisseurs et de leurs caractéristiques radioélectriques (une permittivité, une perméabilité et un coefficient de chiralité). Ces travaux ne sont pas développés ici, pour plus de détail se reporter aux références $[5,8]$.

Nous nous intéressons ici uniquement à des écrans monocouches de type Dallenbach. En effet, la simplicité du dispositif permet de faire ressortir les propriétés des milieux chiraux essentielles à la furtivité par rapport à d'autres plus secondaires. L'outil numérique (modèle MTWC) développé dans la partie précédente sera donc utilisé pour prédire le coefficient de réflexion d'une couche chirale déposée sur un plan métallique.

4.1. Fonctionnement et intérêts. - Dans le formalisme employé (Eqs. (1) et (2)), on peut d'abord noter que le coefficient de réflexion $R_{c c}$ (en incidence normale) pour un écran chiral déposé sur un plan métallique ne dépend pas du coefficient de chiralité $\chi_{c}[6,28]$.

$$
R_{\mathrm{cc}}=\frac{\eta_{0}-Z_{\mathrm{s}}}{\eta_{0}+Z_{\mathrm{s}}}
$$

$\eta_{0}$ est l'impédance du vide et $Z_{\mathrm{s}}$ est l'impédance d'une couche chirale d'épaisseur e (appelée parfois impédance de surface) donnée, dans le cas des équations constitutives de Lindell-Sihvola [2], par :

$$
Z_{\mathrm{s}}=-j \sqrt{\mu_{\mathrm{c}} / \varepsilon_{\mathrm{c}}} \tan \left\{e \omega \sqrt{\varepsilon_{\mathrm{c}} \mu_{\mathrm{c}}}\right\}
$$

En incidence oblique, le coefficient de chiralité rentre explicitement dans le calcul du coefficient de réflexion, mais son influence reste néanmoins négligeable tant que :

$$
\chi_{\mathrm{c}}^{2} \ll \varepsilon_{\mathrm{c}} \mu_{\mathrm{c}}
$$

En utilisant d'autres formalismes $[1,3]$ on note que la chiralité macroscopique a une influence sur ces grandeurs. Par exemple, si l'on utilise le formalisme de Post [1], l'impédance de surface s'écrit :

$$
Z_{\mathrm{s}}=-3 \sqrt{\frac{\mu_{\mathrm{p}}}{\varepsilon_{\mathrm{p}}+\mu_{\mathrm{p}} \xi_{\mathrm{p}}^{2}}} \tan \left\{e \omega \sqrt{\mu_{\mathrm{p}}\left(\varepsilon_{\mathrm{p}}+\mu_{\mathrm{p}} \xi_{\mathrm{p}}^{2}\right)}\right\}
$$

$Z_{\mathrm{s}}$ dépend alors du coefficient de chiralité $\xi_{\mathrm{p}}$, mais dans ce cas, les permittivité $\varepsilon_{\mathrm{p}}$ et perméabilité $\mu_{\mathrm{p}}$ effectives ont des significations énergétiques différentes des paramètres du formalisme de Lindell-Sihvola [6]. Quoi qu'il en soit, les composites à inclusions métalliques chirales de tailles macroscopiques que nous étudions ne créent pas suffisamment de chiralité et l'inégalité (28) est donc toujours vérifiée. Il en résulte que quel que soit le formalisme employé, le coefficient de chiralité (ajustable avec la nature, la géométrie des hélices et la concentration) n'est en aucun cas, un degré de liberté supplémentaire pour satisfaire $R_{\mathrm{cc}}=0$, comme certains auteurs le laissent supposer $[29,30]$. 

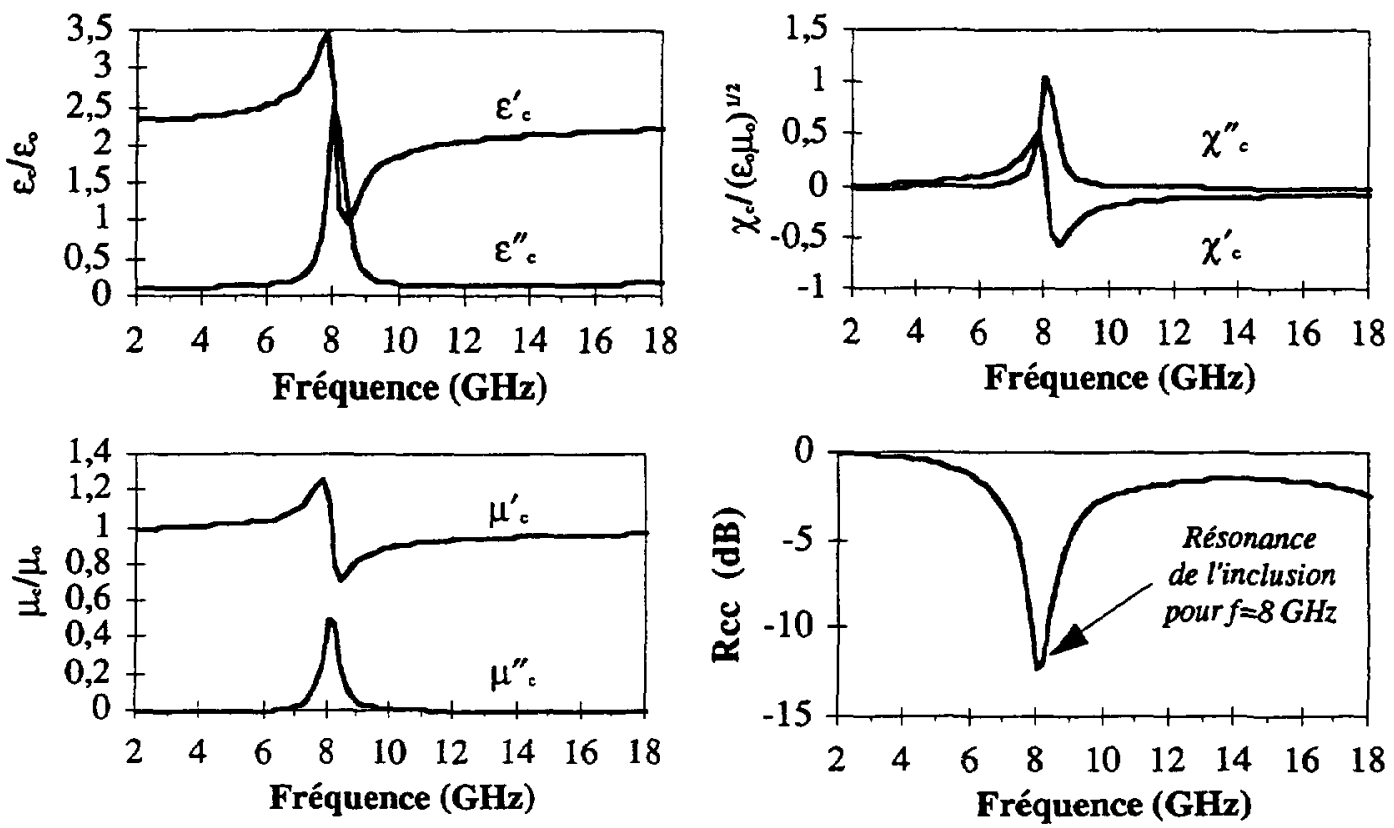

Fig. 12. - Influence de la fréquence de résonance propre des inclusions chirales sur les paramètres effectifs relatifs du composite chiral et sur le coefficient de réflexion (devant court-circuit) : les résultats sont obtenus avec le modèle MTWC. Caractéristiques des hélices : $R=1,0 \mathrm{~mm}, P a s=1,0 \mathrm{~mm}$, $a=0,1 \mathrm{~mm}, 2$ tours droite et $N=20$ hélices $/ \mathrm{cm}^{3}$. Caractéristiques du liant $: \varepsilon=\varepsilon_{0}(1,8+j 0,1)$ et $\mu=\mu_{0}$. Epaisseur de la couche : $7 \mathrm{~mm}$.

[Typical resonances of chiral objects on the effective relative parameters of a chiral composite and the reflection coefficient from a metal-backed lossy chiral slab (results with MTWC model). Helix dimensions: $R=1.0 \mathrm{~mm}, \mathrm{Pitch}=1.0 \mathrm{~mm}, a=0.1 \mathrm{~mm}, 2$ turns and right-handed. Helix density: 20 helices $/ \mathrm{cm}^{3}$. Host material characteristics: $\varepsilon=\varepsilon_{0}(1.8+j 0.1)$ and $\mu=\mu_{0}$. Slab thickness: $7 \mathrm{~mm}$.]

Pour des applications dans le domaine des absorbants, l'intérêt des matériaux chiraux ne réside pas dans la chiralité, comme on pourrait le penser a priori. En fait l'inclusion chirale est intéressante pour sa forme spécifique qui permet de coupler électromagnétiquement le champ électrique et le champ magnétique. Il va donc y avoir une influence sur les moments dipolaires, sur les polarisabilités et par conséquent sur la permittivité et la perméabilité macroscopiques du milieu. On peut donc considérer que le vrai degré de liberté supplémentaire pour réaliser des absorbants est lié à la possibilité de jouer sur les paramètres $\varepsilon_{c}$ et $\mu_{\mathrm{c}}$ et d'adapter l'impédance $Z_{\mathrm{s}}$ à l'interface de manière à obtenir $R_{c c}=0$. Les pics de réflectivités minimales (Fig. 12) sont donc dus à une combinaison adéquate de $\varepsilon_{\mathrm{c}}$ et $\mu_{\mathrm{c}}$. On observe en particulier des zones d'adaptation autour des fréquences de résonance propre de l'objet $(\S 3.1)$.

Sur la figure 13, on a comparé la réponse en réflexion d'un composite contenant des objets chiraux (qui possédent à la fois des propriétés électriques et magnétiques) avec un matériau contenant des motifs identiques constitués d'un objet à propriétés électriques (fibre) et d'un objet à propriétés magnétiques (boucle fermée). On note que l'objet chiral pour un encombrement identique va résonner à fréquence plus basse. La forme enroulée et plus compacte des inclusions chirales peut donc être considérée comme une qualité supplémentaire.

Cet exemple (Fig. 13) permet également d'envisager la manière d'utiliser ces composites pour la furtivité. Lorsque l'on utilise les motifs non chiraux (dont la fréquence de résonance est 


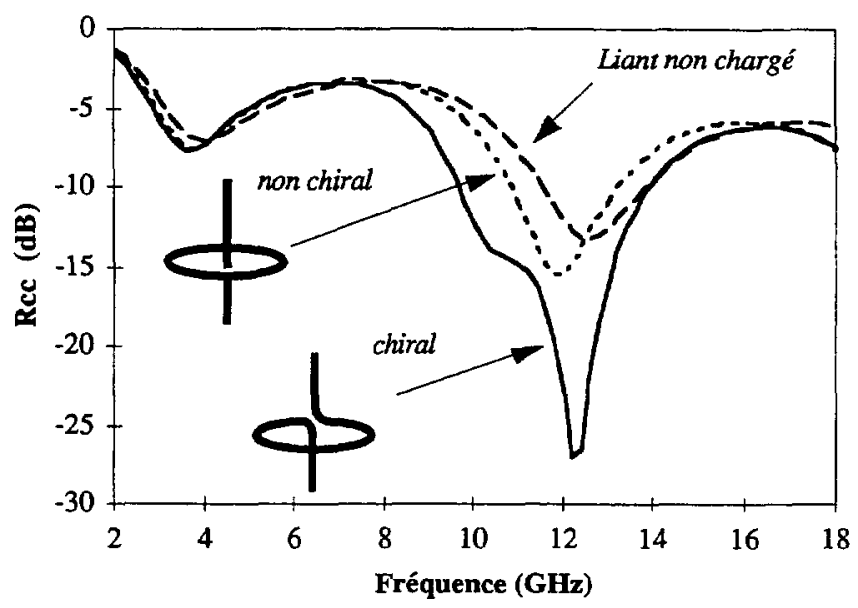

Fig. 13. - Comparaison entre composites contenant des inclusions chirales et non chirales (14 inclusions $/ \mathrm{cm}^{3}$ ). Caractéristiques des hélices modèles : $R=1,05 \mathrm{~mm}, L=0,9 \mathrm{~mm}, a=0,3$ rad et $a=0,1 \mathrm{~mm}$. Caractéristiques des fibres : $L=1,8 \mathrm{~mm}$ et $a=0,1 \mathrm{~mm}$. Caractéristiques des boucles fermées : $R=1,05 \mathrm{~mm}$ et $a=0,1 \mathrm{~mm}$. Caractéristiques du liant $: \varepsilon=\varepsilon_{0}\left(\frac{4,4}{f^{0,25}}+\jmath \frac{1,5}{f^{0,5}}\right)$ où $f$ est en $\mathrm{GHz}$ et $\mu=\mu_{0}$. Epaisseur de la couche : $12 \mathrm{~mm}$.

[Comparison of composites with chiral and non-chiral inclusions (14 inclusions $/ \mathrm{cm}^{3}$ ): results with MTWC model. Canonical helix dimensions: $R=1.05 \mathrm{~mm}, L=0.9 \mathrm{~mm}, a=0.1 \mathrm{~mm}, a=0.3 \mathrm{rad}$. Wire dimensions: $L=1.8 \mathrm{~mm}$ and $a=0.1 \mathrm{~mm}$. Closed loop dimensions: $R=1.05 \mathrm{~mm}$ and $a=0.1$ $\mathrm{mm}$. Host material characteristics: $\varepsilon=\varepsilon_{0}\left(\frac{4.4}{f^{025}}+j \frac{1.5}{f^{05}}\right)$ with $f$ expressed in GHz and $\mu=\mu_{0}$. Slab thickness: $12 \mathrm{~mm}$.]

supérieure à $18 \mathrm{GHz}$ ), on obtient une légère augmentation des paramètres $\varepsilon_{c}, \mu_{c}$ qui va simplement déplacer la résonance d'épaisseur vers les basses fréquences. Dans le cas des inclusions chirales, la résonance propre des hélices s'ajoute à la résonance d'épaisseur et l'on peut ainsi élargir la bande de fréquence où le coefficient de réflexion reste inférieur à $-10 \mathrm{~dB}$ (on passe ainsi de $2,3 \mathrm{GHz}$ avec le cas non chiral à $4,2 \mathrm{GHz}$ avec le composite chiral).

4.2. PerformanCes théoriques et limitations. - La plupart des matériaux chiraux ont été réalisés jusqu'à présent dans un seul but : comprendre les phénomènes physiques intervenant dans l'interaction d'une onde électromagnétique avec un composite hétérogène à structures chirales. De ce fait, les performances obtenues à l'heure actuelle sont limitées. On mentionnera tout de même les composites chiraux à base d'hélices en polymère conducteur réalisés par le CEN Saclay [26]. L'utilisation d'hélices présentant des pertes intrinsèques laisse entrevoir une nouvelle voie de matériaux chiraux absorbants.

Devant le peu de données expérimentales de matériaux absorbants performants et disposant d'un modèle suffisamment fiable, nous étudions différents concepts de composites chiraux ayant théoriquement des propriétés absorbantes intéressantes. Cette étude n'est que préliminaire et ne s'inspire en aucun cas d'une démarche de type "optimisation". Par exemple sur la figure 14, les hélices sont noyées dans une mousse à pertes diélectriques. Les coefficients de réflexion devant court-circuit de la mousse seule (traits pointillés) et de l'écran chiral (trait plein) sont représentés. A masse surfacique quasiment égale (la masse rajoutée par les inclu- 


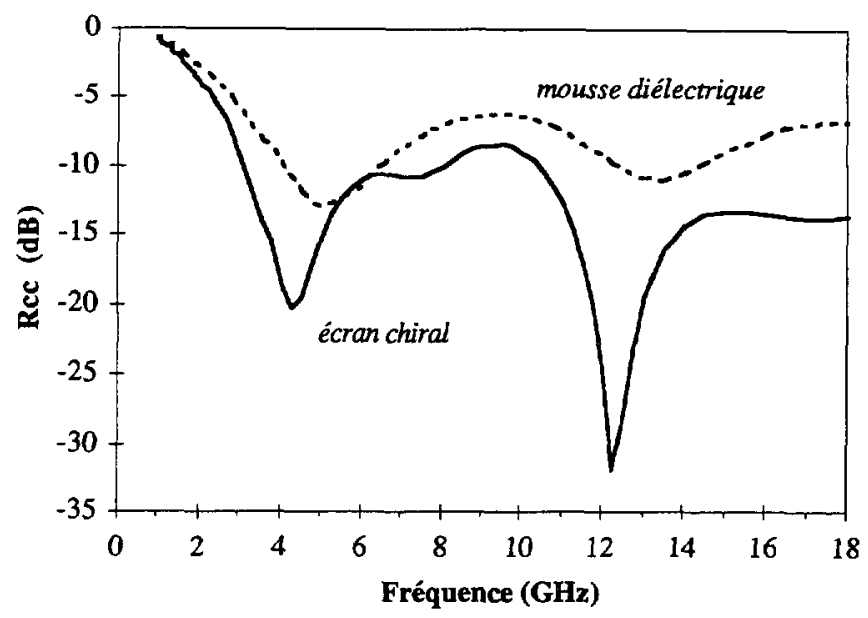

Fig. 14. - Simulation avec le modèle MTWC d'un composite à base d'hélices métalliques : comparaison avec une mousse diélectrique achirale. Caractéristiques des hélices : $R=2,5 \mathrm{~mm}, \mathrm{Pas}=2,1 \mathrm{~mm}$, $a=0,1 \mathrm{~mm}, 1$ tour droite et $N=3,5$ hélices $/ \mathrm{cm}^{3}$. Epaisseur de la couche chirale $: 15 \mathrm{~mm}$.

[Modelling of a chiral slab with randomly oriented regular perfectly conducting helices: comparison with a dielectric achiral absorbing foam. Helix dimensions: $R=2.5 \mathrm{~mm}, P$ itch $=2.1 \mathrm{~mm}, a=0.1 \mathrm{~mm}$, 1 turn and right-handed. Helix density: 3.5 helices $/ \mathrm{cm}^{3}$. Slab thickness: $15 \mathrm{~mm}$.]

sions est négligeable), les performances en terme d'atténuation et de largeur de bande ont été considérablement améliorées par rapport au matériau initial : pratiquement $-10 \mathrm{~dB}$ à partir de $3 \mathrm{GHz}$. Pour créer des zones d'absorption plus étendue en fréquence, on peut envisager d'introduire dans le matériau des objets de tailles différentes. En ajustant les fréquences de résonance de chaque inclusion, on va pouvoir jouer sur la forme du coefficient de réflexion. La figure 15 illustre ce propos avec un composite contenant deux types d'hélices. On observe ainsi une superposition des pics d'absorption créés par chaque catégorie d'inclusion. On note également un élargissement de la bande passante par rapport aux composites ne contenant qu'un seul type d'inclusion.

A travers ces divers exemples théoriques, on voit que les matériaux chiraux peuvent apporter une contribution intéressante dans la conception de matériaux absorbants légers. $A$ priori, il ne sera pas possible d'obtenir des absorbants très minces en partie à cause de la taille des inclusions (quelques millimètres) qu'il faut répartir de façon aléatoire si l'on désire obtenir des composites isotropes. Les études paramétriques montrent que les inclusions fonctionnent mieux dans un liant à faible indice, ce qui permet d'envisager des masses surfaciques relativement faibles. Les simulations ont aussi montré que l'utilisation de substrat à plus fort indice induisait des réflexions importantes sur le premier dioptre (interface air-milieu chiral).

4.3. Conclusions - Perspectives. - Le fonctionnement des matériaux chiraux absorbants semble être compris $[6,28]$ : les pics de réflectivités minimales sont dus à l'influence des inclusions chirales sur les paramètres effectifs $\varepsilon_{\mathrm{c}}$ et $\mu_{\mathrm{c}}$ (adaptation de l'impédance de surface $Z_{\mathrm{s}}$ de la couche chirale), et non à la valeur du coefficient de chiralité nacroscopique $\chi_{\mathrm{c}}$.

Les premiers résultats théoriques en terme d'atténuation montrent que les matériaux chiraux peuvent se révéler intéressants dans la conception de matériaux absorbants légers, fonctionnant à haute fréquence $(>1 \mathrm{GHz})$, mais à fortes épaisseurs. Le choix des différents constituants 


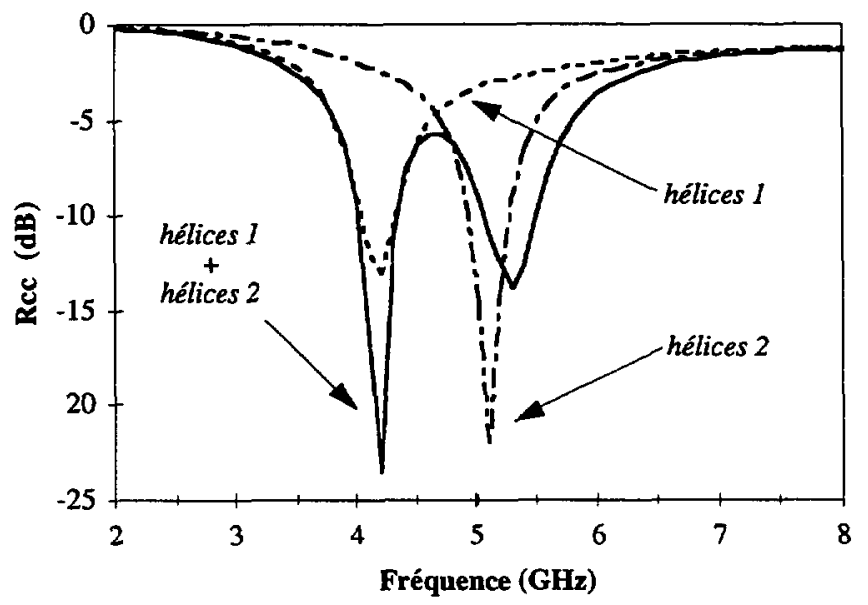

Fig. 15. - Simulation avec le modèle MTWC d'un composite à base d'hélices métalliques de différentes tailles: comparasson avec les composites mono-inclusions. Caractéristiques des hélices de type $1: R=$ $1 \mathrm{~mm}$, Pas $=0,66 \mathrm{~mm}, a=0,1 \mathrm{~mm}, 3$ tours à droite et $N=12$ hélices $/ \mathrm{cm}^{3}$. Caractéristiques des hélices de type 2: $R=0,75 \mathrm{~mm}$, Pas=0,50 mm, $a=0,1 \mathrm{~mm}, 3,5$ tours à droite et $N=12$ hélices $/ \mathrm{cm}^{3}$. Le matériau à inclusions multiples contient 24 hélices $/ \mathrm{cm}^{3}$ (12 de chaque type). Caractéristiques du liant : $\varepsilon=\varepsilon_{0}(2,8+j 0,18)$ et $\mu=\mu_{0}$. Epaisseur de la couche chirale $=15 \mathrm{~mm}$.

[Modelling of a chiral slab with randomly oriented regular perfectly conducting helices of different sizes: comparison with single size inclusion composites. Helix dimensions (type 1): $R=1 \mathrm{~mm}$, Pitch $=0.66 \mathrm{~mm}, a=0.1 \mathrm{~mm}, 3$ turns, right-handed and helix density of 12 helices $/ \mathrm{cm}^{3}$. Helix dimensions (type 2): $R=0.75 \mathrm{~mm}$, Pitch $=0.50 \mathrm{~mm}, a=0.1 \mathrm{~mm}, 3.5$ turns, right-handed and helix density of 12 helices $/ \mathrm{cm}^{3}$. The concentration for the slab containing helices of different sizes is $N=24$ helices $/ \mathrm{cm}^{3}$ (12 of each type). Host material characteristics: $\varepsilon=\varepsilon_{0}(2.8+j 0.18)$ and $\mu=\mu_{0}$. Slab thickness: $15 \mathrm{~mm}$.]

intervenant dans une structure absorbante chirale (permettant de réduire le coefficient de réflexion sur une bande de fréquence souhaitée, pour une incidence et une polarisation données et enfin compatible avec les contraintes d'épaisseur et de poids imposées à l'absorbant) sera difficile et nécessitera une démarche s'inspirant des méthodes d'optimisation pour définir la nature, la géométrie et les dimensions des inclusions chirales, mais aussi la nature du liant.

Ce domaine souffre encore d'un manque d'échantillons de matériaux chiraux. Un effort est donc nécessaire dans la réalisation et les études technologiques de ces nouveaux composites. L'idée d'utiliser des hélices de tailles différentes ou possédant des pertes intrinsèques (polymère conducteur, céramique...) semble être une voie prometteuse et mérite donc d'être cxplorée théoriquement et technologiquement.

\section{Conclusions}

Nous disposons donc d'un modèle numérique (modèle MTWC) qui nous permet de modéliser les propriétés effectives de matériaux hétérogènes à inclusions métalliques de géométrie filaire. Ce modèle a été développé pour étudier plus particulièrement le comportement radiocilectrique des composites chiraux, les inclusions étant dans ce cas des hélices à un ou plusieurs tours.

Notre démarche théorique a été progressivement comparée à des résultats expérimentaux 
ainsi qu'à d'autres modèles. La concordance entre ces différents résultats permet de valider le modèle MTWC et donc d'admettre qu'il est possible de modéliser les composites chiraux par une théorie de milieu effectif tant que la concentration en inclusions reste faible.

Les matériaux chiraux artificiels, ainsi modélisés, peuvent être employés dans la conception d'écrans absorbant les ondes électromagnétiques. Le fonctionnement et les performances de tels écrans ont été étudiés et analysés. Leur optimisation, pour un niveau d'atténuation donné dans un domaine de fréquences défini, reste toutefois très délicate.

Il paraît intéressant d'étudier les composites chiraux à plus fortes concentrations (ce qui revient à étudier l'interaction entres les hélices) pour voir si les modèles développés restent valables et, le cas échéant, travailler sur des théories plus sophistiquées comme les modèles de multidiffusion.

Enfin, il conviendra d'étendre le modèle MTWC à la modélisation des matériaux chiraux à base d'hélices céramiques, composites qui font l'objet aujourd'hui d'un nombre croissant d'études [31].

\section{Annexe A}

Résolution de l'équation variationnelle donnant les courants sur un objet filaire

Il s'agit donc de résoudre l'équation variationnelle suivante :

$$
\begin{aligned}
& \left.\int_{\Gamma} \int_{\Gamma} G\left(\left|r-r^{\prime}\right|\right)\left\{j \omega \mu \mathbf{J}_{\mathrm{s}}\left(r^{\prime}\right) \cdot \mathbf{J}^{\prime}(r)\right)-\frac{j}{\omega \varepsilon}\left(\nabla \cdot \mathbf{J}_{\mathrm{s}}\left(r^{\prime}\right)\right)\left(\nabla \cdot \mathbf{J}^{\prime}(r)\right)\right\} \mathrm{d} r \mathrm{~d} r^{\prime}= \\
& -\int_{\dot{\Gamma}} \mathbf{E}_{\text {inc }}(r) \cdot \mathbf{J}^{\prime}(r) \mathrm{d} r+\int_{\Gamma} Z_{\mathrm{c}} \mathbf{J}_{\mathrm{s}}(r) \cdot \mathbf{J}^{\prime}(r) \mathrm{d} r
\end{aligned}
$$

L'approximation filaire permet d'écrire le courant en coordonnées cylindriques $(\rho, \theta, s)$ sous la forme :

$$
\mathbf{J}_{\mathrm{s}}(s, \theta)=\left|\mathbf{J}_{\mathrm{s}}(s)\right| \boldsymbol{\tau}(\mathrm{s})=J_{\mathrm{s}}(s) \boldsymbol{\tau}(s)
$$

où $\tau(s)$ est le vecteur unitaire porté par $\Sigma$ (Fig. 2a).

Lorsqu'on calcule la distance entre deux points $M(s, \theta)$ et $M^{\prime}\left(s^{\prime}, \theta^{\prime}\right)$ sur $\Gamma$, deux cas peuvent alors se présenter :

- soit $M$ et $M^{\prime}$ sont éloignés l'un par rapport à l'autre; dans ce cas, nous supposons que la distance $\mathrm{d}\left(M, M^{\prime}\right)$ est indépendante $\operatorname{de} \theta$ et $\operatorname{de} \theta^{\prime}, \mathrm{d}$ 'où :

$$
\mathrm{d}\left(M, M^{\prime}\right) \cong\left|M(s)-M\left(s^{\prime}\right)\right|=\sqrt{\left|s-s^{\prime}\right|^{2}}
$$

- soit $M$ et $M^{\prime}$ sont suffisamment rapprochés pour que l'on puisse supposer qu'ils appartiennent à la surface d'un même cylindre rectiligne (Fig. 2b), dans ce cas :

$$
\mathrm{d}\left(M, M^{\prime}\right)=\left|M(s, \theta)-M\left(s^{\prime}, \theta^{\prime}\right)\right| \cong \sqrt{\left|s-s^{\prime}\right|^{2}+4 a^{2} \sin ^{2}\left[\left(\theta-\theta^{\prime}\right) / 2\right]}
$$


La surface $\Gamma$ est paramétrée par $s$ et $\theta, \Gamma=\Sigma \times[0,2 \pi]_{\theta}$. La formule variationnelle (A-1) devient :

$$
\begin{aligned}
& \int_{\Sigma} \int_{\Sigma} \int_{0}^{2 \pi} \int_{0}^{2 \pi} \frac{\exp \{j \beta r\}}{4 \pi r}\left\{j \omega \mu J_{\mathrm{s}}\left(s^{\prime}\right) J^{\prime}(s)\left(\tau\left(s^{\prime}\right) \cdot \tau^{\prime}(s)\right)-\frac{j}{\omega \varepsilon} \frac{\mathrm{d} J_{\mathrm{s}}\left(s^{\prime}\right)}{\mathrm{d} s^{\prime}} \frac{\mathrm{d} J^{\prime}(s)}{\mathrm{d} s}\right\} a^{2} \mathrm{~d} \theta \mathrm{d} \theta^{\prime} \mathrm{d} s \mathrm{~d} s^{\prime} \\
& =-\int_{\Sigma} \int_{0}^{2 \pi} \mathbf{E}_{\mathrm{inc}}(s) \cdot \tau^{\prime}(s) J^{\prime}(s) a \mathrm{~d} s \mathrm{~d} \theta+\int_{\Sigma} \int_{0}^{2 \pi} Z_{c} J_{\mathrm{s}}(s) J^{\prime}(s) a \mathrm{~d} \theta
\end{aligned}
$$

$r$ étant défini par : $r=\sqrt{\left(\left|s-s^{\prime}\right|^{2}+4 a^{2} \sin ^{2}\left[\left(\theta-\theta^{\prime}\right) / 2\right]\right.}$

En remarquant que $r\left(\left|s-s^{\prime}\right|,\left(\theta-\theta^{\prime}\right) / 2\right)$ est $2 \pi$ périodique en $\theta$ pour $\theta^{\prime}$ fixé, le noyau $G$ ou fonction de Green peut s'écrire :

$$
\begin{aligned}
G(u)= & \int_{0}^{2 \pi} \int_{0}^{2 \pi} \frac{\exp \left\{j \beta r\left(u,\left(\theta-\theta^{\prime}\right) / 2\right)\right\}}{4 \pi r\left(u,\left(\theta-\theta^{\prime}\right) / 2\right)} \mathrm{d} \theta \mathrm{d} \theta^{\prime}=2 \int_{0}^{2 \pi} \frac{\exp \{j \beta r(u, v)\}}{4 \pi r(u, v)} \mathrm{d} v \\
& \text { avec } u=\left|s-s^{\prime}\right| \in[0, L] \text { et } v=\left(\theta-\theta^{\prime}\right) / 2
\end{aligned}
$$

On montre que ce noyau se décompose en une partie à singularité logarithmique $G_{\mathrm{S}}$ et une partie régulière $G_{\mathbf{R}}$ :

$$
\begin{aligned}
G_{\mathrm{s}}(u) & =\frac{\exp \{\jmath \beta u\}}{a} \ln \left(\frac{1}{u}\right) \\
G_{\mathrm{R}}(u) & =2 \int_{0}^{\pi / 2} \frac{\exp \{j \beta r(u, v)\}-\exp \{j \beta u\} \cos (v)}{r(u, v)} \mathrm{d} v \\
& +\frac{\exp \{j \beta u\}}{a} \ln \left(2 a+\sqrt{u^{2}+4 a^{2}}\right)
\end{aligned}
$$

Lorsque la double intégration en $\theta$ et $\theta^{\prime}$ pour le noyau de Green est effectuée, il reste à résoudre la double intégrale sur $\Sigma \times \Sigma$ pour les inconnues $J_{\mathrm{s}}$ et $J^{\prime}$. Cette équation est la suivante :

$$
\begin{aligned}
& \int_{\Sigma} \int_{\Sigma} G\left(\left|s-s^{\prime}\right|\right)\left\{j \omega \mu J_{\mathrm{s}}\left(s^{\prime}\right) J^{\prime}(s)\left(\tau\left(s^{\prime}\right) \cdot \tau^{\prime}(s)\right)-\frac{j}{\omega \varepsilon} \frac{\mathrm{d} J_{\mathrm{s}}\left(s^{\prime}\right)}{\mathrm{d} s^{\prime}} \frac{\mathrm{d} J^{\prime}(s)}{\mathrm{d} s}\right\} a \mathrm{~d} s \mathrm{~d} s^{\prime} \\
= & -\int_{\Sigma} \int_{0}^{2 \pi} \mathbf{E}_{\mathrm{inc}}(\mathrm{s}) \cdot \boldsymbol{\tau}^{\prime}(\mathrm{s}) J^{\prime}(s) \mathrm{d} s \mathrm{~d} \theta+\int_{\Sigma} \int_{0}^{2 \pi} Z_{\mathrm{c}} J_{\mathrm{s}}(s) \mathrm{d} s \mathrm{~d} \theta
\end{aligned}
$$

avec le noyau $G=G_{\mathrm{S}}+G_{\mathrm{R}}$. La solution de l'équation (A-8) est recherchée sous la forme :

$$
J_{\mathrm{s}}(\mathrm{s})=\sum_{\jmath=1}^{N} J_{\jmath} \Phi_{\jmath}(s)
$$

Les fonctions $\Phi_{3}$ sont des polynômes de degré 1 qui servent également de fonctions tests. Ainsi si l'on définit les coefficients $a_{\imath \jmath}$ et $b_{\imath}$ comme :

$$
\begin{aligned}
a_{\imath \jmath}= & \int_{\Sigma} \int_{\Sigma} G\left(\left|s-s^{\prime}\right|\right)\left\{j \omega \mu \Phi_{\jmath}\left(s^{\prime}\right) \Phi_{\imath}(s) \cos \left(\tau\left(s^{\prime}\right), \tau(s)\right)\right. \\
& \left.-\frac{j}{\omega \varepsilon} \frac{\mathrm{d} \Phi_{\jmath}\left(s^{\prime}\right)}{\mathrm{d} s^{\prime}} \frac{\mathrm{d} \Phi_{\imath}(s)}{\mathrm{d} s}\right\} a \mathrm{~d} s \mathrm{~d} s^{\prime}-\int_{\Sigma} \int_{0}^{2 \pi} Z_{\mathrm{c}} \Phi_{\jmath}(s) \Phi_{\imath}(s) \mathrm{d} s \mathrm{~d} \theta
\end{aligned}
$$




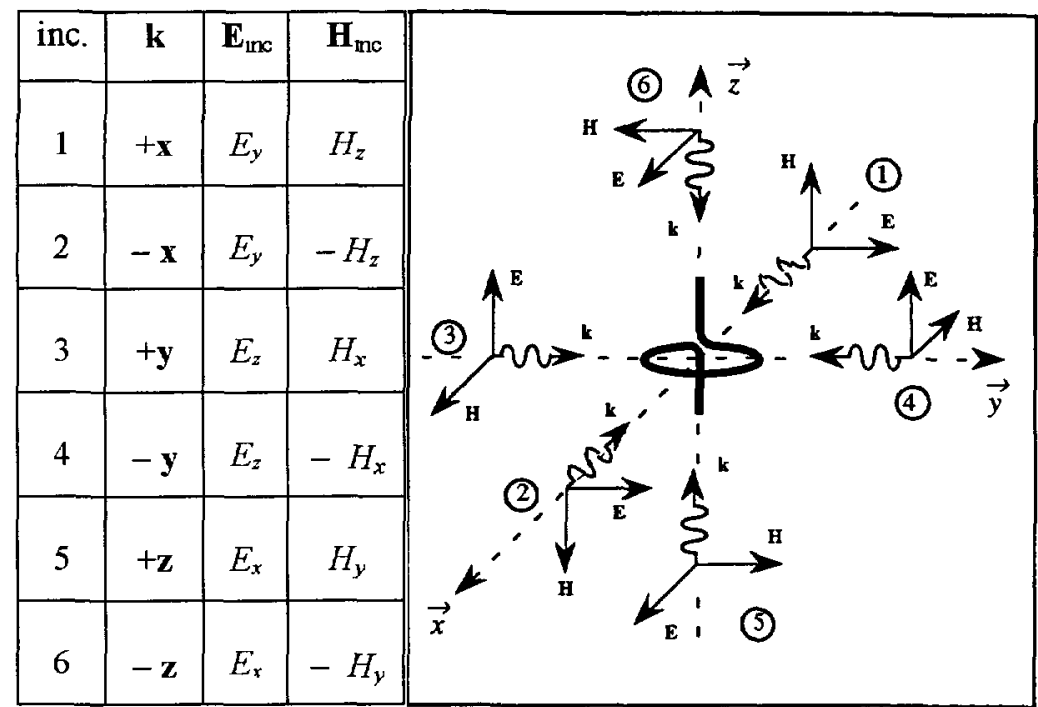

Fig. 16. - Incidences nécessaires à la détermination des tenseurs de polarisabilités d'une hélice. $\mathbf{k}$ représente le vecteur d'onde de l'onde électromagnétique incidente $\left(\mathbf{E}_{\mathrm{inc}}, \mathbf{H}_{\mathrm{inc}}\right)$.

[Incident waves necessary at the calculation of polarizability tensors of small bi-anisotropic scatterers. $\mathbf{k}$ is the wave vector of the incident electromagnetic wave $\left(\mathbf{E}_{\mathrm{nnc}}, \mathbf{H}_{\mathrm{nc}}\right)$.]

$$
b_{\imath}=-\int_{\Sigma} \int_{0}^{2 \pi}\left(\mathbf{E}_{\mathrm{inc}}(s) \cdot \boldsymbol{\tau}^{\prime}(s)\right) \Phi_{\imath}(s) \mathrm{d} s \mathrm{~d} \theta
$$

on peut définir la matrice $A$ et les vecteurs $J$ et $B$

$$
A=\left\{a_{2}\right\}, \quad B=\left\{b_{2}\right\} \quad \text { et } \quad J=\left\{J_{2}\right\}
$$

et la résolution du système matriciel $A J=B$ permet d'obtenir le courant $J_{\mathrm{s}}$ en tout point de l'objet filaire.

\section{Annexe B}

\section{Détermination des tenseurs de polarisabilités}

Pour déterminer les tenseurs de polarisabilités d'une hélice, on calcule les vecteurs $\mathbf{p}$ et $\mathbf{m}$ pour les six incidences d'onde qui sont présentées sur la figure 16. Les moments dipolaires sont obtenus en intégrant les courants à la surface de l'hélice donnés par la version filaire du code ARLENE. Le choix particulier de ces six incidences permet d'obtenir de manière simple des équations dont les inconnues sont les éléments des tenseurs. Prenons comme exemple les cas des incidences 1 et 2 qui conduisent aux équations suivantes et permettent de calculer 12 composantes :

$$
\left[\begin{array}{l}
\left(p_{1 x}+p_{2 x}\right) / 2 \\
\left(p_{1 y}+p_{2 y}\right) / 2 \\
\left(p_{1 z}+p_{2 z}\right) / 2
\end{array}\right]=\varepsilon\left[\begin{array}{lll}
- & \alpha_{\mathrm{e}_{12}} & - \\
- & \alpha_{\mathrm{e}_{22}} & - \\
- & \alpha_{\mathrm{e}_{32}} & -
\end{array}\right]\left[\begin{array}{c}
0 \\
E_{y} \\
0
\end{array}\right]
$$




$$
\begin{aligned}
& {\left[\begin{array}{c}
\left(p_{1 x}-p_{2 x}\right) / 2 \\
\left(p_{1 y}-p_{2 y}\right) / 2 \\
\left.p_{1 z}-p_{2 z}\right) / 2
\end{array}\right]=\varepsilon \eta\left[\begin{array}{ll}
- & - \\
- & \alpha_{\mathrm{em}_{13}} \\
- & \alpha_{\mathrm{em}_{23}} \\
- & \alpha_{\mathrm{em}_{23}}
\end{array}\right]\left[\begin{array}{c}
0 \\
0 \\
H_{z}
\end{array}\right]} \\
& {\left[\begin{array}{c}
\left(m_{1 x}+m_{2 x}\right) / 2 \\
\left(m_{1 y}+m_{2 y}\right) / 2 \\
\left(m_{1 z}+m_{2 z}\right) / 2
\end{array}\right]=\frac{1}{\eta}\left[\begin{array}{ll}
- & \alpha_{\mathrm{me}_{12}} \\
- & \alpha_{\mathrm{me}_{22}} \\
- & \alpha_{\mathrm{me}_{32}}
\end{array}\right]\left[\begin{array}{c}
0 \\
E_{y} \\
0
\end{array}\right]}
\end{aligned}
$$

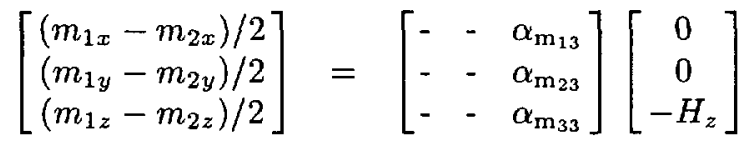

Il suffit ensuite de réitérer le processus pour les incidences 3-4 et 5-6. L'étude des six cas de polarisation va donc permettre d'obtenir assez d'équations pour pouvoir calculer les 36 inconnues qui composent les différents tenseurs. La nature réciproque des objets diffractants ainsi modélisés fait que les tenseurs de susceptibilité vérifient les relations suivantes :

$$
\overline{\bar{\alpha}}_{\mathrm{e}}=\overline{\bar{\alpha}}_{\mathrm{e}}^{\mathrm{T}} \quad \overline{\bar{\alpha}}_{\mathrm{m}}=\overline{\bar{\alpha}}_{\mathrm{m}}^{\mathrm{T}} \quad \overline{\bar{\alpha}}_{\mathrm{me}}=-\overline{\bar{\alpha}}_{\mathrm{em}}^{\mathrm{T}}
$$

où T désigne la transposée du tenseur.

\section{Remerciements}

Les auteurs remercient vivemement Frédéric Guérin (IRCOM et THOMSON CSF-LCR), Xavier Lafosse (CEA/Saclay) et Alain Froger (CEA/CER) pour les données expérimentales sur les matériaux chiraux qu'ils ont réalisés. Ils remercient également Daniel Gogny, Bertrand Remy, Damien Clementz, Pascal Jesné (CEA/CESTA) ainsi qu'Isabelle Proust, pour la relecture de ce manuscrit et les nombreuses discussions fructueuses.

\section{Bibliographie}

[1] Jaggard D.L., Mickelson A.R et Papas C.H., On Electromagnetic Waves in Chiral Media, Appl. Phys. 18 (1979) 211-216.

[2] Sihvola A.H. et Lindell I.V., Bi-Isotropic Constitutive Relations, Microwave Opt Technol Lett 4 (1991) 295-297.

[3] Lakhtakia A., Varadan V.K. et Varadan V.V., Time Harmonic Electromagnetic Fields in Chiral Media, Lectures Notes in physics $n^{\circ} 335$ (Springer Verlag, Berlin, 1989).

[4] Kong J.A., Theorems of Bianisotropic Media, Proc. IEEE 60 (1972) 1036-1046.

[5] Engheta N. et Jaggard D.L., Electromagnetic Chirality and Its Applications, IEEE Antennas Propag. Soc. Newslett. 30 (1988) 6-12.

[6] Guérin F., Energy dissipation and absorption in bi-isotropic media described by different formalism, PIER 9, Special Issue on Biisotropic Media and Applications, A. Priou et J. A. Kong Eds. (Elsevier, New York, 1994) pp. 31-44.

[7] Zoudhi S., Fourrier Lamer A. et Mariotte F., On the relationships between the constitutives parameters of chiral materials and dimensions of chiral objects (helix), J. Phys. III France 2 (1992) 337-343.

[8] Mariotte F., Matériaux chiraux à structures hétérogènes : modélisations et applications, Thèse de Doctorat de l'Université de Bordeaux I (Mars 1994). 
[9] Sihvola A.H. and Lindell I.V., Chiral Maxwell Garnett Mixing Formula, Electron. Lett. 26 (1990) 118-119.

[10] Tretyakov S.A., Mariotte F., Simovski K.R., Kharina T.G., et Héliot J.P., Analytical antenna model for chiral scatterers: comparison with numerical and experimental data, soumis à la revue IEEE Trans. Antennas Propag. (1994).

[11] Mariotte F., Gogny D., Bourgeade A. et Farail F., Backscattering of the thin wire helix: analytical model, numerical study and free space measurements. Application to chiral composite modelling, Proc. Bianisotropics'93, Gomel (October 1993).

[12] Mariotte F., Tretyakov S.A. et Sauviac B., Isotropic chiral composites modeling: comparison with analytical, numerical and experimental results, Microwave Opt. Technol. Lett. 7 (1994) 861-864.

[13] Mariotte F., Guérin F., Bourgeade A. et Bannelier P., Numerical computations of the electromagnetic field scattered by complex chiral shapes, J. Electromagnetıc Waves and Applicatıons, à paraitre en 1995.

[14] F. Mariotte et J.P. Parneix Eds., Proc. of 3rd International Workshop on Chiral, Bi-isotropic and Bi-anisotropic Media, Chiral'94 (Périgueux, May 1994).

[15] Guérin F., Mariotte F., Leroy B. et Bannelier P., Modeling chiral composites at CEA-CESTA and Thomson-CSF: from RCS to effective properties computation, Proc. of 3rd International Workshop on Chiral, Bi-isotropic and Bi-anisotropic Media, Chiral'94 (Périgueux, May 1994) pp. 59-69.

[16] Oberschmidt G. et Jacob A.F., On the resonant behaviour of chiral materials, Proc. of 24thEuMC (Cannes, September 1994) pp. 372-377.

[17] Brewitt-Taylor C.R., Modelling of helix-loaded chiral radar absorbing layers, PIERS Proc., 259 (July 1993, Pasadena, USA).

[18] Whites K.W., Host effects on the constitutive parameters for synthetic chiral media, Proc. of 3rd International Workshop on Chiral, Bi-isotropic and Bi-anisotropic Media, Chiral'94 (Périgueux, May 1994) pp. 77-82.

[19] Svigelj J., Michielssen E. et Mittra R., Numerical studies of the wire helix, IEEE AP-S International Symposium, (Chicago, 1992) pp. 695-698.

[20] Varadan V.V, Varadan V.K., Terosky J. et Quarry M., EM wave propagation in composites containing multiply connected inclusions, Proc. of 3rd International Workshop on Chiral, Bi-isotropic and Bi-anisotropic Media, Chiral'94 (Périgueux, May 1994) pp. 95.

[21] Robin T., Souillard B., Accolas R., Theret P. et Bolioli S., Modelling of electromagnetic properties of helix-loaded chiral media, Proc. of 3rd International Workshop on Chiral, Bi-isotropic and Bianisotropic Media, Chiral'94 (Périgueux, May 1994) pp. 103-108.

[22] Artola M., Effective properties of some chiral composite media, Proceedings of 3rd International Workshop on Chiral, Bi-isotropic and Bi-anisotropic Media, Chiral'94 (Périgueux, May 1994) pp. 97-102.

[23] Bourgeade A., Gay J., Héliot J.P., Leroy B. et Mariotte F., The integral equation code "ARLENE": application to the numerical simulation of chiral inclusions, Proc. of 3rd International Workshop on Chiral, Bi-isotropic and Bi-anisotropic Media, Chiral'94 (Périgueux, May 1994) pp. $267-284$.

[24] Knott E.F.. Schaeffer J.F. et Tuley M.T., Radar Cross Section, Second Edition - Artech House (1993) pp. 13-21.

[25] Jaggard D.L. et Sun X., Theory of Chiral Multilayers, J. Opt. Soc. Am. 9 (1992) 804-813.

[26] Lafosse X., Preparation of new chiral composite with conductive polymers and free space characterization, Proc. of Progress in Electromagnetics Research Symposium (PIERS'94) (Noordwijk, 1994).

[2i] Froger A., Bourgeoisat A., Garat J., Bardy N. et Kroll Y., Caractérisation d'un matériau composite chiral, Actes des 3èmes Journées sur la Caractérisation Microonde des Matériaux, session V-09 (Brest, Octobre 1994). 
[28] Brewitt-Taylor C.R., "Modelling of helix-loaded chiral radar absorbing layers", PIER 9, Special Issue on Bïsotropic Media and Applications, A. Priou et J. A. Kong Eds. (Elsevier, New York) (1994) 289-310.

[29] Jaggard D.L. et Engheta N., Chirosorb ${ }^{\text {TM }}$ as an invisible media, Electron. lett. 25 (1989) 173-174.

[30] Jaggard D.L., Engheta N. et Liu J.C., Chiroshield ${ }^{\text {TM }}$ a Salisbury / Dallenbach shield alternative, Electron. lett. 26 (1990) 1332-1333.

[31] Guérin F., Microwave chiral materials: a review of experimental studies and some results on composites with ferroelectric inclusions, PIER 9, Special Issue on Birsotropic Media and Applications, A. Priou et J. A. Kong Eds. (Elsevier, New York, 1994) pp. 219-263. 